$\underline{\text { Preprint typeset in JHEP style - HYPER VERSION }}$

hep-th/0611296

KCL-MTH-06-12

NSF-KITP-06-110

\title{
Reflection and Transmission for Conformal Defects
}

\author{
Thomas Quella \\ KdV Institute for Mathematics, University of Amsterdam, Plantage Muidergracht 24, \\ 1018 TV Amsterdam - NL \\ E-mail: tquella@science.uva.nl \\ Ingo Runkel \\ Department of Mathematics, King's College London, Strand, London WC2R 2LS - UK \\ E-mail: Ingo.Runkel@kcl.ac.uk \\ Gérard M.T. Watts \\ Department of Mathematics, King's College London, Strand, London WC2R 2LS - UK \\ E-mail: Gerard.Watts@kcl.ac.uls
}

\begin{abstract}
We consider conformal defects joining two conformal field theories along a line. We define two new quantities associated to such defects in terms of expectation values of the stress tensors and we propose them as measures of the reflectivity and transmissivity of the defect. Their properties are investigated and they are computed in a number of examples. We obtain a complete answer for all defects in the Ising model and between certain pairs of minimal models. In the case of two conformal field theories with an enhanced symmetry we restrict ourselves to non-trivial defects that can be obtained by a coset construction.
\end{abstract}

Keywords: Conformal and W Symmetry, Boundary Quantum Field Theory. 


\section{Contents}

1. Introduction 2

2. Reflection and transmission coefficients 3

2.1 Definition of $\mathcal{R}$ and $\mathcal{T}$

$2.2 \mathcal{R}, \mathcal{T}$ and topological defects

3. The free boson 8

4. The Ising model and the free fermion 9

4.1 The Ising model

4.2 Free fermions 15

5. Conformal defects from coset decompositions 16

5.1 Transmission of a common sub-symmetry 17

5.2 An example for the general case 18

6. Minimal models with rational products 23

6.1 Lee-Yang $\times$ Lee-Yang 24

6.2 Lee-Yang $\times$ Ising 24

6.3 Lee-Yang $\times \mathbf{M}_{2,7}$

7. Conclusions and outlook 26

A. Reflection and Transmission via $\omega_{b} \quad 29$

B. Summary on states in the coset decomposition 30

G. Category theoretic calculations 31

C.1 Preliminaries 32

C.1.1 Induced modules 32

C.1.2 Local modules and factorising defects 32

C.1.3 Computing the coefficient $\omega$

C.1.4 Action of topological defects 34

C.2 Lee-Yang $\times$ Lee-Yang 35

C.3 Lee-Yang $\times$ Ising 37

C.4 Lee-Yang $\times \mathbf{M}_{2,7}$ 


\section{Introduction}

Just as a conformal boundary condition describes a universality class of boundary critical behaviour in a two-dimensional quantum system, a conformal defect is a universality class of critical behaviour at a one-dimensional junction of two such quantum systems. It is therefore of some interest to understand the properties of conformal defects, and consequently there exist numerous publications emphasising a variety of different aspects. To name just a few, there has been considerable effort to clarify the role of defects and impurities in concrete applications in statistical models [1, 2] (see also references therein),

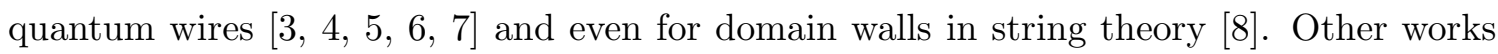
focused on general constructive methods [9, 10, 11, 12, 13, 14] or structural implications [15, 16, 17]. Finally, there are also articles which have originally been written in a different context but have implications for the study of defect systems [18, 19, 20, 21, 22].

In order to set the stage, let us consider a conformally invariant quantum system on the complex plane, which is inhomogeneous in the sense that the theory on the upper half-plane is described by a conformal field theory $\mathrm{CFT}_{1}$, and on the lower half-plane by a conformal field theory $\mathrm{CFT}_{2}$, possibly with a different chiral symmetry or even a different value of the central charge. The two CFTs meet along the real line, which constitutes a defect where the fields of the two theories can have discontinuities or divergences. If the defect has the property that inside every correlator

$$
\lim _{y \rightarrow 0}\left(T^{1}(x+i y)-\bar{T}^{1}(x+i y)\right)=\lim _{y \rightarrow 0}\left(T^{2}(x-i y)-\bar{T}^{2}(x-i y)\right) \quad \text { for all } x \in \mathbb{R},
$$

where $T^{1,2}$ and $\bar{T}^{1,2}$ are the holomorphic and anti-holomorphic components of the stress tensor of $\mathrm{CFT}_{1,2}$, then the defect is called conformal. There are two special solutions to the condition (1.1). Firstly, the two sides of (1.1) can individually be zero. Then the real line is a conformal boundary to $\mathrm{CFT}_{1}$ and $\mathrm{CFT}_{2}$ separately and the two theories are decoupled; such conformal defects are called 'totally reflective' or 'factorising'. Second, it can be that on the real line we have $T^{1}(x)=T^{2}(x)$ and $\bar{T}^{1}(x)=\bar{T}^{2}(x)$ so that the defect is invisible to correlators of the stress tensor. Such defects are called 'totally transmissive' or 'topological'. The latter type of conformal defect can only exist if the central charges of the two theories coincide. The properties of topological defects in rational conformal field theories have been studied in detail in [9, 12, 23, 15, 16, 17.

The classification of a complete set of conformal defects joining two given CFTs is a very difficult problem, just as is that of finding all conformal boundary conditions. However, even for Virasoro minimal models, where all conformal boundary conditions can be constructed [24], apart from a few exceptions (see sections 4 and 6), it is not known how to obtain all conformal defects. The only systematic investigation of conformal defects in minimal models which extend beyond pure transmission or reflection has been performed in the Ising case [1, 2]. For a single free boson, on the other hand, just one specific oneparameter family of conformal defects has been discussed in [8]. The situation is slightly better in models with an enhanced chiral symmetry such as WZW models or coset theories. Here one can apply a (nested) coset construction [13] (see also 20, 25] for a more explicit treatment of groups and cosets) to systematically reduce the symmetry preserved 
by the defect. A different class of non-factorising defects for WZW and coset models is conjectured to arise from 'permutation-like' boundary conditions in product CFTs. So far however, the analysis of this type of defects has either been restricted to the semi-classical regime 21] or to models which allow one to use insights from topological conformal field theories [22, 26, 27].

Given this variety of constructions it is helpful to have a simple quantity at one's disposal which is relatively easy to compute and contains some basic information about the conformal defect. One such quantity is the $g$-function [28], which can be defined by relating the conformal defect to a conformal boundary condition in the folded model (see section 2). We propose, also in section 2, two additional such quantities $\mathcal{R}$ and $\mathcal{T}$ (related via $\mathcal{R}+\mathcal{T}=1$ ), defined in terms of expectation values of the stress tensors of $\mathrm{CFT}_{1}$ and $\mathrm{CFT}_{2}$. Their properties suggest they might be useful quantitative indicators of the reflectivity and transmissivity of the conformal defect. This is motivated by the fact that $\mathcal{R}=1$ for totally reflective defects and $\mathcal{R}=0$ for totally transmissive defects, as well as $\mathcal{R}+\mathcal{T}=1$. In sections 3 6 we proceed to compute $\mathcal{R}$ and $\mathcal{T}$ for a selection of conformal defects in certain CFTs. The explicit models we consider are the free boson, the Ising model, defects between WZW theories arising from the nested coset construction, and those pairs of Virasoro minimal models which have a product that is again a minimal model. For two general rational CFTs we describe, in section 5.1, defects that are transmissive only with respect to a common rational sub-symmetry (which does not necessarily contain the Virasoro algebras of the two CFTs). Altogether, we find that in the unitary examples treated, $\mathcal{R}$ and $\mathcal{T}$ take values in the interval $[0,1]$, while in non-unitary theories they can violate these bounds. The bulk of the technical computations has been gathered in several appendices.

\section{Reflection and transmission coefficients}

\subsection{Definition of $\mathcal{R}$ and $\mathcal{T}$}

While it is possible to describe conformal defects as operators between the Hilbert space of one CFT and another, it is more usual to treat them as boundary conditions in an enlarged theory obtained by 'folding' the lower half-plane to lie on top of the upper half-plane to

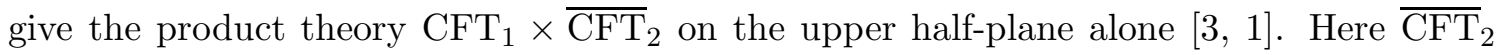
stands for the theory obtained by exchanging holomorphic and anti-holomorphic degrees of freedom in $\mathrm{CFT}_{2} \cdot{ }^{1}$ The real line then is a boundary for $\mathrm{CFT}_{1} \times \overline{\mathrm{CFT}}_{2}$ and condition (1.1) amounts to demanding the boundary condition to be conformal in the sense of [29]. In the folded picture, the topological defects are a special case of so-called 'permutation branes' studied in [19], and factorising defects correspond to boundary conditions in the product theory, for which the boundary state can be written as a product of boundary states for the individual CFTs.

\footnotetext{
${ }^{1}$ Passing from $\mathrm{CFT}_{2}$ to $\overline{\mathrm{CFT}}_{2}$ may involve a choice of convention; the identification of degrees of freedom is only unique up to automorphisms of $\mathrm{CFT}_{2}$. Once such an identification is fixed, the relation between conformal defects linking $\mathrm{CFT}_{1}$ to $\mathrm{CFT}_{2}$ and conformal boundary conditions of $\mathrm{CFT}_{1} \times \overline{\mathrm{CFT}}_{2}$ is uniquely fixed as well.
} 
If we map the upper half-plane in the folded model to the exterior of the unit circle then the boundary is represented by a 'boundary state' $|b\rangle$ in the bulk Hilbert space. This space is the tensor product of the Hilbert spaces of $\mathrm{CFT}_{1}$ and $\overline{\mathrm{CFT}}_{2}$ and the condition (1.1) is

$$
\left(\left(L_{m}^{1}+L_{m}^{2}\right)-\left(\bar{L}_{-m}^{1}+\bar{L}_{-m}^{2}\right)\right)|b\rangle=0 .
$$

It guarantees the existence of an infinite-dimensional conformal symmetry in the defect system.

Conformal boundary conditions (or defects) can be thought of as special cases of integrable boundary conditions (or defects) [30, 31, 32, 33, 34, 35, 36]. Integrable boundary conditions have been mainly considered for massive integrable field theories with a multiparticle spectrum. For such theories an integrable boundary condition (or defect) is almost entirely characterised by a reflection (or reflection/transmission) matrix giving the amplitudes for a single particle hitting the boundary (or defect) to emerge as a particle of a different species. We would like to find a quantity which captures at least some of the information contained in such a matrix in the case of conformal field theories where the particle interpretation is rather involved or missing.

Let us first consider the case of free massless fields. To quote one formula for the free boson (this is reviewed in section 3), the boundary state representing the conformal defect satisfies

$$
\left(a_{m}^{i}-S_{i j} \bar{a}_{-m}^{j}\right)|b\rangle=0,
$$

so that the reflection and transmission amplitudes for the bosonic modes $a_{m}^{i}$ and $\bar{a}_{-m}^{i}$ are constant, given by the matrix $S_{i j}$.

We would like to extend this picture to more general conformal field theories in which a particle interpretation is unknown or complicated. In a general conformal field theory the only tool we have is the Virasoro algebra and consequently it would be good if we could find an analogue of (2.2) involving the Virasoro algebra. If we suppose that

$$
\left(L_{m}^{i}-S_{i j} \bar{L}_{-m}^{j}\right)|b\rangle=0,
$$

then we find that this can only be consistent with the Virasoro algebra for the choices

$$
S=\left(\begin{array}{ll}
1 & 0 \\
0 & 1
\end{array}\right), \quad \text { or } \quad S=\left(\begin{array}{ll}
0 & 1 \\
1 & 0
\end{array}\right),
$$

that is for purely reflecting or purely transmitting defects.

Since we cannot define a matrix $S_{i j}$ by (2.3) for a general conformal defect, we consider instead the matrix

$$
R_{i j}=\frac{\left\langle 0\left|L_{2}^{i} \bar{L}_{2}^{j}\right| b\right\rangle}{\langle 0 \mid b\rangle}
$$

This would be simply related to the matrix $S_{i j}$ if (2.3) held. Using general properties of boundary states, one can show that the form of $R_{i j}$ is fixed up to a single parameter $\omega_{b}$,

$$
R=\frac{c_{1} c_{2}}{2\left(c_{1}+c_{2}\right)}\left[\left(\begin{array}{cc}
\frac{c_{1}}{c_{2}} & 1 \\
1 & \frac{c_{2}}{c_{1}}
\end{array}\right)+\omega_{b}\left(\begin{array}{cc}
1 & -1 \\
-1 & 1
\end{array}\right)\right] .
$$


The proof of this is given in appendix A. Note that considering $\left\langle 0\left|L_{n}^{i} \bar{L}_{n}^{j}\right| b\right\rangle$ in addition to $R_{i j}$ does not give more information. This follows since evaluating $\langle 0| L_{n}^{i} \bar{L}_{n+1}^{j}$ on (2.1) with $m=1$, one obtains a simple recursion relation which leads to

$$
\frac{\left\langle 0\left|L_{n}^{i} \bar{L}_{n}^{j}\right| b\right\rangle}{\langle 0 \mid b\rangle}=\frac{n\left(n^{2}-1\right)}{6} R_{i j} \quad \text { for } n \geq 0 .
$$

The matrix $R_{i j}$ is closely related to the 'entropic admittance matrix' $Y_{S}(\omega)_{i j}$ introduced in [6, 6] to describe the entropy flow in junctions of quantum wires. The explicit relation is obtained by Fourier transform of [0, eqn. (87)], ${ }^{2}$

$$
Y_{S}(\omega)_{i j}=f(\omega)\left(R_{i j}-\frac{c_{i}}{2} \delta_{i j}\right)=f(\omega) \frac{c_{1} c_{2}\left(\omega_{b}-1\right)}{2\left(c_{1}+c_{2}\right)}\left(\begin{array}{cc}
1 & -1 \\
-1 & 1
\end{array}\right)
$$

with $f(\omega)=(\beta /(2 \pi))^{3}\left(k \hbar v^{2}\right)^{2}\left(1+(\hbar \beta \omega /(2 \pi))^{2}\right) / 6$ and $\omega_{b}$ the parameter appearing in (2.6).

Rather than characterising a defect by the value of $\omega_{b}$, we instead propose the following two quantities which have very appealing properties:

$$
\begin{aligned}
& \mathcal{R}=\frac{2}{c_{1}+c_{2}}\left(R_{11}+R_{22}\right)=\frac{c_{1}^{2}+2 c_{1} c_{2} \omega_{b}+c_{2}^{2}}{\left(c_{1}+c_{2}\right)^{2}}, \\
& \mathcal{T}=\frac{2}{c_{1}+c_{2}}\left(R_{12}+R_{21}\right)=\frac{2 c_{1} c_{2}\left(1-\omega_{b}\right)}{\left(c_{1}+c_{2}\right)^{2}} .
\end{aligned}
$$

They satisfy $\mathcal{R}=1$ for purely reflecting defects and $\mathcal{R}=0$ for purely transmitting defects, and together with the obvious relation $\mathcal{R}+\mathcal{T}=1$ this prompts our calling them reflection and transmission coefficients. They also have obvious physical interpretations in the case of certain defects in a single free boson or free fermion theory, where $\mathcal{R}$ and $\mathcal{T}$ are the probabilities of reflection or transmission of the free field modes.

The above definition of $\mathcal{R}$ and $\mathcal{T}$ is in terms of boundary states in the 'folded' theory. We can also define them via the expectation values of the components of the stress-energy tensor on the two sides of the defect. Consider the complex plane with a conformal defect on the real line, and denote by $T^{1}, \bar{T}^{1}$ and $T^{2}, \bar{T}^{2}$ the components of the stress tensor of the CFTs on the two sides of the defect. Then instead of (2.9) we can write

$$
\mathcal{R}=\frac{\left\langle T^{1} \bar{T}^{1}+T^{2} \bar{T}^{2}\right\rangle_{1 \mid 2}}{\left\langle\left(T^{1}+\bar{T}^{2}\right)\left(\bar{T}^{1}+T^{2}\right)\right\rangle_{1 \mid 2}} \quad \text { and } \quad \mathcal{T}=\frac{\left\langle T^{1} T^{2}+\bar{T}^{1} \bar{T}^{2}\right\rangle_{1 \mid 2}}{\left\langle\left(T^{1}+\bar{T}^{2}\right)\left(\bar{T}^{1}+T^{2}\right)\right\rangle_{1 \mid 2}} .
$$

Here, $T^{1}$ and $\bar{T}^{1}$ are inserted at the point $i y$ on the upper half-plane, while $T^{2}$ and $\bar{T}^{2}$ are inserted at the point -iy. Since the numerator and the denominator in these formulas are both proportional to $y^{-4}, \mathcal{R}$ and $\mathcal{T}$ do not depend on the choice of $y$. Note also that, as opposed to the $g$-function, because $\mathcal{R}$ and $\mathcal{T}$ are defined as quotients they are not additive if one considers superpositions of defects.

\footnotetext{
${ }^{2}$ In [7] the focus is on near-critical junctions in bulk-critical quantum wires. We are concerned only with conformal defects, which correspond to critical junctions. While 2.8 obeys properties A,B,D,E,F listed in [7, sect. V], we are not certain how to reconcile it with property C.
} 


\section{$2.2 \mathcal{R}, \mathcal{T}$ and topological defects}

One very useful property of the quantities $\mathcal{R}$ and $\mathcal{T}$ is their invariance under the action of topological defects, which we will now explain.

First note that since the stress tensor is continuous across a topological defect, the defect commutes with local conformal transformations and can be deformed continuously without affecting the value of a correlator. This is the reason for the qualifier 'topological' (introduced in [37]). Now consider two topological defect lines $X$ and $Y$ which are running parallel to each other. Moving them very close together, they look like a new topological defect, which is called the fused defect $X \star Y$. Altogether this defines the fusion ring of topological defects [9, 10, 11, 12]. Let us call a topological defect elementary if it cannot be written as the sum of two other topological defects. Then, even if one starts with two elementary topological defects $X, Y$, the fused defect $X \star Y$ is typically no longer elementary. One can also consider a topological defect $X$ close to a conformal boundary $B$. Since $X$ commutes with the stress tensor, moving the defect against the boundary gives rise to a new conformal boundary condition $X \star B$ [10, 15, 38]. This defines an action of topological defects on boundary conditions. Again, even if $X$ and $B$ are elementary, $X \star B$ is typically not. ${ }^{3}$

In the case where we have $\mathrm{CFT}_{1}$ on the upper half-plane and $\mathrm{CFT}_{2}$ on the lower halfplane, separated by a conformal defect $D$, we can place a topological defect $X$ of $\mathrm{CFT}_{1}$ on the line $\mathbb{R}+i L$, for some $L>0$, and a topological defect $Y$ of $\mathrm{CFT}_{2}$ on the line $\mathbb{R}-i L$. In the limit $L \rightarrow 0$ we obtain a new conformal defect $X \star D \star Y$ (which is in general not elementary even if $X, Y$ and $D$ were). As for conformal boundary conditions, in this way we obtain an action of topological defects on conformal defects.

One can also wonder if it is possible to fuse two parallel conformal defects which are not topological. In this case the correlator does depend on their distance and, much like in the OPE of two fields, one would expect divergences as one takes the distance to zero (see [8] for an explicit calculation). We will not investigate this situation in the present paper.

Consider now the correlator (2.10) defining $\mathcal{R}$, but with topological defects $X$ and $Y$ placed on the lines $\mathbb{R} \pm i L$. As the defects are topological, the resulting correlators will not depend on $L$. Taking $L$ to infinity removes the defects from the complex plane, multiplying the correlator by an overall constant which cancels between the numerator and denominator, and we obtain the quantity $\mathcal{R} \equiv \mathcal{R}(D)$ in the presence of the conformal defect $D$. This is nothing but the procedure of 'inflating a defect in a world sheet' used extensively in 16, 17]. Taking $L$ to zero, which we can do because $X$ and $Y$ commute with the stress tensors, gives rise to the fused conformal defect $X \star D \star Y$. In this way we obtain the identity

$$
\mathcal{R}(D)=\mathcal{R}(X \star D \star Y) \quad \text { for all topological defects } X, Y \text {. }
$$

Of course, the same holds also for $\mathcal{T}$. In other words, $\mathcal{R}$ and $\mathcal{T}$ are functions on the set of conformal defects that are invariant under the action of topological defects.

\footnotetext{
${ }^{3}$ We will use the qualifier 'elementary' for boundary conditions and conformal defects in the same sense as for topological defects.
} 
Note that one can also define a 'universal ground state degeneracy' $g(D)$ for a conformal defect $D$ by considering the corresponding quantity of the conformal boundary condition in the folded model as defined in [28]. However, unless acting with so-called group-like defects (which are topological defects that have an inverse w.r.t. defect fusion), $g(X \star D \star Y)$ will be different from $g(D)$.

Let $D$ be an elementary conformal defect. We say that another elementary conformal defect $D^{\prime}$ is generated from $D$ by the action of topological defects if there are topological defects $X, Y$ such that $D^{\prime}$ occurs in the decomposition of $X \star D \star Y$ into elementary defects. While (2.11) tells us that a weighted average of $\mathcal{R}\left(D^{\prime}\right)$ over all $D^{\prime}$ that occur in the decomposition of $X \star D \star Y$ is equal to $\mathcal{R}(D)$, under certain conditions we even have $\mathcal{R}(D)=\mathcal{R}\left(D^{\prime}\right)$ for all such $D^{\prime}$, as we will now see.

For an elementary conformal defect $D$, let $F=X \star D \star Y$ have the decomposition $F=\sum_{\alpha} D_{\alpha}$ into elementary defects $D_{\alpha}$. We will work in the folded picture, and denote the conformal boundary conditions corresponding to $D, F$, and $D_{\alpha}$ by the same symbols. The topological defects $X$ and $Y$ can be expressed as a single topological defect $X \bar{Y}$ in the product theory $\mathrm{CFT}_{1} \times \overline{\mathrm{CFT}}_{2}$.

First note that by moving only a portion of the topological defect $X \bar{Y}$ to a boundary with boundary condition $D$ we obtain the identity
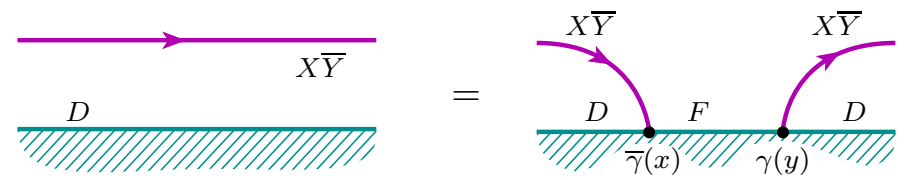

The portion of the defect $X \bar{Y}$ moved to the boundary fuses with the boundary $D$ to become the boundary condition $F$ and the $X \bar{Y}$ defect now ends and starts at the junctions of the boundary conditions $D$ and $F ; \gamma(x)$ and $\bar{\gamma}(y)$ are the (Virasoro-primary weight zero) boundary fields that mark the end- and starting-points of the defect $X \bar{Y}$.

Second, note that the fact that we can decompose $F=\sum_{\alpha} D_{\alpha}$ means that we can find Virasoro-primary weight zero boundary fields $P_{\alpha}$ on $F$ which form a complete set of orthogonal idempotents w.r.t. to the OPE, i.e. $P_{\alpha}(x) P_{\beta}(y)=\delta_{\alpha, \beta} P_{\alpha}(y)$ and $\sum_{\alpha} P_{\alpha}(x)=$ $\mathbf{1}_{F}$, the identity field on $F$. In fact, the $P_{\alpha}(x)$ are just the identity fields for the individual boundary conditions $D_{\alpha}$. For example, a correlator of some bulk fields on a disc with boundary condition $D_{\alpha}$ is equal to a disc correlator with the same bulk fields, but with boundary condition $F$ and an insertion of $P_{\alpha}$ on the boundary.

Let now $\Phi$ be a bulk field of $\mathrm{CFT}_{1} \times \overline{\mathrm{CFT}}_{2}$ that commutes with topological defects of the form $X \bar{Y}$. We have the following equalities of disc correlators,

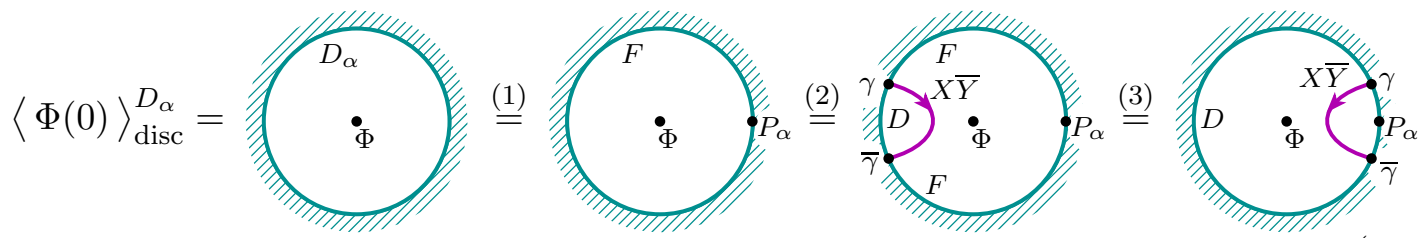

Here in step (1) we replaced the boundary condition $D_{\alpha}$ by $F$ and an insertion of $P_{\alpha}$, in step (2) we used the inverse transformation of (2.12), and in (3) we moved $\gamma$ and $\bar{\gamma}$ close 
to the $P_{\alpha}$ insertion and took the topological defect $X \bar{Y}$ past the bulk field $\Phi$, as we can do by assumption. Suppose now that in addition the following two conditions hold:

(i) Up to scalar multiples, there is a unique Virasoro-highest weight boundary field of weight zero on the $D$ boundary (namely the identity field $\mathbf{1}_{D}$ ).

(ii) The disc partition function with boundary condition $D_{\alpha},\langle\mathbf{1}\rangle_{\text {disc }}^{D_{\alpha}}$, is nonzero.

Then we can conclude further that

$$
\langle\Phi(0)\rangle_{\mathrm{disc}}^{D_{\alpha}}=C_{\alpha, \gamma, \bar{\gamma}}\langle\Phi(0)\rangle_{\mathrm{disc}}^{D}
$$

for some nonzero constant $C_{\alpha, \gamma, \bar{\gamma}}$ which depends on the choice of $\gamma, \bar{\gamma}$ and $\alpha$, but not on the bulk field $\Phi$. This has to be true, since the field one obtains by collapsing the remaining defect bubble in (2.13) has to be Virasoro-primary of weight zero, and by assumption (i) it thus is proportional to the identity field $\mathbf{1}_{D}$; the constant $C_{\alpha, \gamma, \bar{\gamma}}$ appearing in (2.14) is nothing but this proportionality constant. It is nonzero because by assumption (ii) the left hand side of (2.14) is nonzero in the case $\Phi=\mathbf{1}$.

If we insert the identity (2.14) into the definition (2.9) of $\mathcal{R}$ (transformed to the unit disc), we obtain, with the choices $\Phi=T^{1} \bar{T}^{1}+T^{2} \bar{T}^{2}$ and $\Phi=\mathbf{1}$, respectively,

$$
\mathcal{R}\left(D_{\alpha}\right)=\frac{2}{c_{1}+c_{2}} \frac{\left\langle T^{1} \bar{T}^{1}+T^{2} \bar{T}^{2}\right\rangle_{\mathrm{disc}}^{D_{\alpha}}}{\langle\mathbf{1}\rangle_{\mathrm{disc}}^{D_{\alpha}}}=\frac{2}{c_{1}+c_{2}} \frac{C_{\alpha, \gamma, \bar{\gamma}}\left\langle T^{1} \bar{T}^{1}+T^{2} \bar{T}^{2}\right\rangle_{\mathrm{disc}}^{D}}{C_{\alpha, \gamma, \bar{\gamma}}\langle\mathbf{1}\rangle_{\mathrm{disc}}^{D}}=\mathcal{R}(D) .
$$

Let us make two comments. First, our assumption that $D$ is an elementary defect is necessary but not sufficient for condition (i) to hold. Second, the requirement (ii) is not really a restriction, because it is already implicit in the formulation of $\mathcal{R}$ and $\mathcal{T}$. If (ii) did not hold, (2.5) would be ill-defined.

Thus, starting from a defect $D$ obeying (i) and (ii), all defects generated from $D$ have the same value of $\mathcal{R}$. Conversely, one can now ask whether for a given value $\mathcal{R}_{0}$, one can find a preferred defect $D_{0}$ with $\mathcal{R}\left(D_{0}\right)=R_{0}$, such that all elementary conformal defects $D^{\prime}$ with $\mathcal{R}\left(D^{\prime}\right)=\mathcal{R}_{0}$ can be generated by the action of topological defects on $D_{0}$.

For the examples in section 6, where all conformal defects are known, the answer is yes. In the example treated in section 5.2, this is still true for all conformal defects that preserve a certain extended chiral algebra, but we cannot make statements about the behaviour of all conformal defects. For the Ising model (section 1) it is true for the defects with discrete excitation spectrum (so that there is an unambiguous notion of 'elementary defect'); it would however not hold true for the exceptional defects about which we speculate at the end of that section.

\section{The free boson}

The simplest model in which conformal defects have been studied is a single free scalar boson which was investigated in [8]. In that paper the defect was placed vertically in the plane and the scalar field to the left and right of the defect were denoted $\phi^{1}$ and $\phi^{2}$ 
respectively and related at the defect by

$$
\left(\begin{array}{l}
\partial_{-} \phi^{1} \\
\partial_{+} \phi^{2}
\end{array}\right)=S\left(\begin{array}{l}
\partial_{+} \phi^{1} \\
\partial_{-} \phi^{2}
\end{array}\right)
$$

where $S$ is either of the two matrices

$$
S=\left(\begin{array}{cc}
-\cos (2 \theta) & \sin (2 \theta) \\
\sin (2 \theta) & \cos (2 \theta)
\end{array}\right), \quad S^{\prime}=\left(\begin{array}{cc}
\cos (2 \theta) & -\sin (2 \theta) \\
\sin (2 \theta) & \cos (2 \theta)
\end{array}\right) .
$$

In the folded picture the boundary state representing this defect is

$$
\begin{aligned}
|b\rangle & =\mathcal{N} \prod_{n=1}^{\infty} \exp \left(\frac{1}{n} a_{-n}^{i} \bar{a}_{-n}^{j} S_{i j}\right)|0\rangle \\
& =\mathcal{N}\left(1+a_{-1}^{i} \bar{a}_{-1}^{j} S_{i j}+\frac{1}{2}\left(a_{-1}^{i} \bar{a}_{-1}^{j} S_{i j}\right)^{2}+a_{-2}^{i} \bar{a}_{-2}^{j} S_{i j}+\ldots\right)|0\rangle
\end{aligned}
$$

where $a_{n}^{i}$ and $\bar{a}_{n}^{i}$ are the modes of $\phi^{i}$ and $\mathcal{N}$ is a normalisation constant. The modes themselves are normalised such that $\left[a_{m}, a_{n}\right]=m \delta_{m+n, 0}$. Since $c_{1}=c_{2}=1$ and the energy-momentum tensors are of the standard form,

$$
L_{-2}^{i}|0\rangle=\frac{1}{2} a_{-1}^{i} a_{-1}^{i}|0\rangle,
$$

it is easy to calculate

$$
\left\langle 0\left|L_{2}^{i} \bar{L}_{2}^{j}\right| b\right\rangle=\frac{\mathcal{N}}{2}\left(S_{i j}\right)^{2}
$$

so that for both cases 3.2

$$
\left\langle 0\left|L_{2}^{\mathrm{tot}} \bar{L}_{2}^{\mathrm{tot}}\right| b\right\rangle=\frac{\mathcal{N}}{2} \sum_{i, j}\left(S_{i j}\right)^{2}=\mathcal{N},
$$

and

$$
\mathcal{R}=\cos ^{2}(2 \theta), \quad \mathcal{T}=\sin ^{2}(2 \theta) .
$$

These are the reflection and transmission probabilities for the massless modes in this model. Note that the quantity $\mathcal{R}$ we have defined is not the same quantity as the $\mathcal{R}$ calculated in [8] which is the reflection amplitude for a massless mode.

\section{The Ising model and the free fermion}

The name of Ising model is given to various different theories with $c=1 / 2$ : the theory of purely local fields of the modular invariant theory; the local theory of the free fermion; the non-local theory obtained by combining the two. In section 4.1 we discuss the defects in the modular invariant theory and in section 4.2 the defects in the free fermion and their

relation to those in the modular invariant theory. From here on, when we refer to "the Ising model" we shall always mean the modular invariant theory. 


\subsection{The Ising model}

Conformal defects in the Ising model have been studied exhaustively by Oshikawa and Affleck in [1, 2]. ${ }^{4}$ The Ising model has central charge $c=1 / 2$ so that the doubled model used in the folded treatment of conformal defects has central charge one. Oshikawa and Affleck use two different identifications of this $c=1$ model, firstly as a special case of the Ashkin-Teller model and secondly as a particular case of the orbifolded free boson. We shall use only this second identification for our calculations. We review briefly this construction and then calculate the reflection and transmission coefficients for the conformal defects they have found. Throughout this section we use the notation of [1, 2].

The doubled Ising model can be identified with the $r=1$ orbifolded free boson, that is a free boson which classically takes values in the line segment $[0, \pi]$.

It is usual to construct the orbifolded free boson starting from a free boson compactified on a circle. A complete classification of the conformal boundary conditions for the free boson on a circle has been proposed by Friedan [41] with some more details given by Janik [42] and Gaberdiel and Recknagel [43]. Since the radius 1 is not a rational multiple of the self-dual radius $r=1 / \sqrt{2}$, according to 41 the conformal boundary conditions split into three classes: a circle of Dirichlet boundary conditions on the free boson, a circle of Neumann boundary conditions, and a line segment of boundary conditions which break the $U(1)$ symmetry of the free boson. The construction of the orbifolded free boson boundary states from those of the free boson on a circle is straightforward for the first two classes; the third class we will return to later.

The Dirichlet boundary conditions on the orbifolded free boson $\varphi$ are $\varphi=\varphi_{0} \in[0, \pi]$ and the Neumann boundary conditions can be expressed in terms of the dual field $\tilde{\varphi}$ as $\tilde{\varphi}=\tilde{\varphi}_{0} \in[0, \pi / 2]$. These boundary conditions are elementary except at the end points where the presence of twisted sectors splits them into two. This leads to the following space of boundary conditions:

$$
\begin{gathered}
D_{O}\left(\varphi_{0}\right) \text { with } \varphi_{0} \in(0, \pi), \quad D_{O}(0)_{ \pm}, \quad D_{O}(\pi)_{ \pm} \\
N_{O}\left(\tilde{\varphi}_{0}\right) \text { with } \tilde{\varphi}_{0} \in(0, \pi / 2), \quad N_{O}(0)_{ \pm}, \quad N_{O}(\pi / 2)_{ \pm} .
\end{gathered}
$$

To construct the space of states we first describe the space of ground states of the unorbifolded free boson on a circle of radius 1 . These are labelled by two integers $(m, n)$ and denoted $|(m, n)\rangle$; the winding number is $m$ and the total momentum is $n$ and the conformal dimensions of such a state are $h=\left(m+\frac{1}{2} n\right)^{2} / 2, \bar{h}=\left(-m+\frac{1}{2} n\right)^{2} / 2$. On these ground states the oscillators have integer modes. In the twisted sector the oscillators have half-integer modes and the twisted ground states are $|0\rangle_{T}$ and $|\pi\rangle_{T}$ with conformal dimension 1/16.

The calculation of $\omega$ can be reduced to finding the overlap of the boundary state with a particular bulk state, $|W \bar{W}\rangle$, as explained in appendix A. The form of the primary state $|W \bar{W}\rangle$ can be deduced from consideration of the Ashkin-Teller formulation in which it is primary of weight $(2,2)$ with respect to the total Virasoro algebra and a descendent of

\footnotetext{
${ }^{4}$ From a lattice or spin chain perspective, the critical properties of the Ising model with defect lines have also been studied earlier, see e.g. [39, 40] and the references in [1], 2. 2]. For our analysis we only need the boundary states in the folded model as first given by Oshikawa and Affleck.
} 
the product of the identity representations of the two $c=1 / 2$ Virasoro algebras under the action of the two separate Virasoro algebras. In [2], such a primary state is denoted by $|2, I I\rangle$ and also by $|2,1\rangle$. From a comparison of the boundary states of the Ising $\times$ Ising model constructed in [2], in the Ashkin-Teller and free boson formulations, the form of the state $|2, I I\rangle$ in the orbifolded free boson construction can be identified, and fixing the normalisation as in A.1 we get

$$
|W \bar{W}\rangle=\frac{1}{8}|2, I I\rangle=\frac{1}{16}(|(0,4)\rangle+|(0,-4)\rangle+|(2,0)\rangle+|(-2,0)\rangle) .
$$

Calculating $\omega$ and using the formulae $\mathcal{R}=(1+\omega) / 2, \mathcal{T}=(1-\omega) / 2$ we get from (2.9) in this case, we find for the various boundary conditions:

$$
\begin{array}{c|ccc} 
& \omega & \mathcal{R} & \mathcal{T} \\
\hline D_{O}\left(\varphi_{0}\right) & \cos \left(4 \varphi_{0}\right) & \cos ^{2}\left(2 \varphi_{0}\right) & \sin ^{2}\left(2 \varphi_{0}\right) \\
N_{O}\left(\tilde{\varphi}_{0}\right) & \cos \left(4 \tilde{\varphi}_{0}\right) & \cos ^{2}\left(2 \tilde{\varphi}_{0}\right) & \sin ^{2}\left(2 \tilde{\varphi}_{0}\right)
\end{array}
$$

Note that these also hold true at the end points; the twisted sectors make no difference to the values of $\omega, \mathcal{R}$ or $\mathcal{T}$.

The purely transmitting defects correspond to the Ising model topological defects as identified in [1, 2] - the trivial or identity defect is $\mathbb{1}=D_{O}(\pi / 4)$, the spin-reversal is $\varepsilon=D_{O}(3 \pi / 4)$ and the duality defect is $\sigma=N_{O}(\pi / 4)$.

The purely reflecting defects correspond to pairs of boundary conditions in the two copies of the Ising model. We find a small difference from the identification given in [2], in that the Neumann factorising boundary conditions have left and right factors swapped. If we label the three boundary conditions of the Ising model as,+- and $f$ for spin up, spin down and free, then the identification of the reflecting defects in the doubled model is:

\begin{tabular}{c|c|c|c|c|c|c|c|c}
$(++)$ & $(--)$ & $(-+)$ & $(+-)$ & $(f f)$ & $(f+)$ & $(f-)$ & $(+f)$ & $(-f)$ \\
\hline$D_{O}(0)_{+}$ & $D_{O}(0)_{-}$ & $D_{O}(\pi)_{+}$ & $D_{O}(\pi)_{-}$ & $D_{O}(\pi / 2)$ & $N_{O}(0)_{+}$ & $N_{O}(0)_{-}$ & $N_{O}\left(\frac{\pi}{2}\right)_{+}$ & $N_{O}\left(\frac{\pi}{2}\right)_{-}$
\end{tabular}

This can be checked by computing the overlaps of the boundary states or by calculating the form of the topological defects in the two copies of the Ising model and their actions on the boundary states. Since this seems of some interest, we give the details here.

The topological defects in CFTs $A$ and $B$ on either side of a conformal defect are also topological defects in the product theory $A \times \bar{B}$ in which the conformal defect is represented by a boundary state. We identify these 'product defects' in the case of Ising $\times$ Ising by writing down the general ansatz for a topological defect and fixing the coefficients by computing the action on the factorising boundary states (representing factorising defects).

We do not need to construct the whole topological defect operator - only that part which has non-zero action on the boundary states we are interested in. The boundary states are linear combinations of Virasoro Ishibashi states, one for each spinless primary state (those states which are highest weight for both the left and right Virasoro algebras with $h=\bar{h}$ ) which are given in [2]. We repeat the list here but giving the primary states 
in the free boson form rather than the Ashkin-Teller notation in [2]. For $n \in \mathbb{Z}_{\geq 0}$,

\begin{tabular}{c|c}
$h=\bar{h}$ & Highest weight states \\
\hline$n^{2}$ & $|n\rangle^{\text {Vir }}$ \\
$\frac{1}{2}(n+1)^{2}$ & $\frac{1}{\sqrt{2}}(|(0,2 n+2)\rangle+|(0,-2 n-2)\rangle), \frac{1}{\sqrt{2}}(|(n+1,0)\rangle+|(-n-1,0)\rangle)$ \\
$\frac{1}{8}(2 n+1)^{2}$ & $\frac{1}{\sqrt{2}}(|(0,2 n+1)\rangle+|(0,-2 n-1)\rangle)$ \\
$\frac{1}{16}(2 n+1)^{2}$ & $|n\rangle_{0, T}^{\text {Vir }},|n\rangle_{\pi, T}^{\text {Vir }}$
\end{tabular}

The states $|n\rangle^{\text {Vir }}$ are highest weight combinations of free boson modes on the untwisted vacuum and $|n\rangle_{\varphi_{0}, T}^{\mathrm{Vir}}$ are highest weight combinations of free boson modes on the twisted vacua, for example

$$
|1\rangle^{\operatorname{Vir}}=a_{-1} \bar{a}_{-1}|0\rangle,|1\rangle_{\pi, T}^{\operatorname{Vir}}=a_{-1 / 2} \bar{a}_{-1 / 2}|\pi\rangle_{T} .
$$

We will write the projectors onto the Virasoro representations with $h$ equal to $n^{2}$ and $(2 n+1)^{2} / 8$ as $P_{n}$ and $Q_{n}$ respectively, and the matrix part of the topological defect acting on the representations of weight $(n+1)^{2} / 2$ in the basis (4.5) as

$$
\left(\begin{array}{ll}
a & b \\
c & d
\end{array}\right)_{n}
$$

Finally, the matrix part of the topological defect mixing the twisted sectors in the basis (4.5) we write as

$$
\left(\begin{array}{ll}
a & b \\
c & d
\end{array}\right)_{n, T}
$$

Thus, we consider defects of the form

$$
\hat{X}=\sum_{n=0}^{\infty}\left(a_{n} P_{n}+b_{n} Q_{n}+\left(\begin{array}{cc}
c_{n} & d_{n} \\
e_{n} & f_{n}
\end{array}\right)_{n}+\left(\begin{array}{ll}
g_{n} & h_{n} \\
j_{n} & k_{n}
\end{array}\right)_{n, T}\right)+\text { other terms }
$$

where 'other terms' are parts which annihilate the conformal boundary states.

One can deduce from [2] or from the character formulae for the vacuum and twisted free boson representations that the free boson Dirichlet and Neumann Ishibashi states are given in terms of the Virasoro Ishibashi states as follows:

$$
\begin{array}{rlrl}
|(0,0)\rangle\rangle_{D} & \left.=\sum_{n=0}^{\infty}|n\rangle\right\rangle^{\mathrm{Vir}}, & |\phi\rangle\rangle_{T, D} & \left.=\sum_{n=0}^{\infty}|n\rangle\right\rangle_{\phi, T}^{\mathrm{Vir}}, \\
\mid(0,0))\rangle_{N} & \left.=\sum_{n=0}^{\infty}(-1)^{n}|n\rangle\right\rangle^{\mathrm{Vir}}, & \left.|\phi\rangle\rangle_{T, N}=\sum_{n=0}^{\infty}(-1)^{n(n+1) / 2}|n\rangle\right\rangle_{\phi, T}^{\mathrm{Vir}} .
\end{array}
$$


where $\phi \in\{0, \pi\}$ labels the twisted sector. The identification of the factorised boundary conditions in the Dirichlet sector fixes the forms of the identity and $\varepsilon$ defects as

$$
\begin{aligned}
\mathbb{1}_{L}=\mathbb{1}_{R} & =\sum_{n=0}^{\infty}\left(P_{n}+Q_{n}+\left(\begin{array}{ll}
1 & 0 \\
0 & 1
\end{array}\right)_{n}+\left(\begin{array}{ll}
1 & 0 \\
0 & 1
\end{array}\right)_{n, T}\right)+\ldots \\
\varepsilon_{L} & =\sum_{n=0}^{\infty}\left(P_{n}-Q_{n}+\left(\begin{array}{ll}
1 & 0 \\
0 & 1
\end{array}\right)_{n}+\left(\begin{array}{ll}
0 & 1 \\
1 & 0
\end{array}\right)_{n, T}\right)+\ldots \\
\varepsilon_{R} & =\sum_{n=0}^{\infty}\left(P_{n}-Q_{n}+\left(\begin{array}{ll}
1 & 0 \\
0 & 1
\end{array}\right)_{n}+\left(\begin{array}{cc}
0 & -1 \\
-1 & 0
\end{array}\right)_{n, T}\right)+\ldots
\end{aligned}
$$

These satisfy $\varepsilon_{L}^{2}=\varepsilon_{R}^{2}=1$, and the total spin reversal, given as the product of the reversals in the two sectors,

$$
\varepsilon_{L} \varepsilon_{R}=\sum_{n=0}^{\infty}\left(P_{n}+Q_{n}+\left(\begin{array}{ll}
1 & 0 \\
0 & 1
\end{array}\right)_{n}+\left(\begin{array}{cc}
-1 & 0 \\
0 & -1
\end{array}\right)_{n, T}\right)+\ldots
$$

does just reverse the sign of the twisted sector contributions as noted in [1], 2]

To complete the set of topological defects, we find the duality defects are

$$
\begin{aligned}
\sigma_{L} & =\sqrt{2} \sum_{n=0}^{\infty}\left((-1)^{n} P_{n}+\left(\begin{array}{ll}
0 & 1 \\
1 & 0
\end{array}\right)_{n}+\frac{1}{2}(-1)^{n(n+1) / 2}\left(\begin{array}{ll}
1 & 1 \\
1 & 1
\end{array}\right)_{n, T}\right)+\ldots \\
\sigma_{R} & =\sqrt{2} \sum_{n=0}^{\infty}\left((-1)^{n} P_{n}+(-1)^{n}\left(\begin{array}{ll}
0 & 1 \\
1 & 0
\end{array}\right)_{n}+\frac{1}{2}(-1)^{n(n+1) / 2}\left(\begin{array}{cc}
1 & -1 \\
-1 & 1
\end{array}\right)_{n, T}\right)+\ldots
\end{aligned}
$$

Again it is easy to check that these obey the correct algebra:

$$
\sigma_{L}^{2}=\mathbb{1}+\varepsilon_{L}, \quad \sigma_{R}^{2}=\mathbb{1}+\varepsilon_{R}, \quad \sigma_{L} \varepsilon_{L}=\sigma_{L}, \quad \sigma_{R} \varepsilon_{R}=\sigma_{R}
$$

We can now investigate the action of the topological defects on the Dirichlet and Neumann line of boundary conditions in the orbifolded model:

$$
\begin{array}{ll}
\varepsilon_{L}\left|D_{O}(\varphi)\right\rangle=\varepsilon_{R}\left|D_{O}(\varphi)\right\rangle=\left|D_{O}(\pi-\varphi)\right\rangle, & \varepsilon_{L}\left|N_{O}(\tilde{\varphi})\right\rangle=\varepsilon_{R}\left|N_{O}(\tilde{\varphi})\right\rangle=\left|N_{O}(\tilde{\varphi})\right\rangle \\
\sigma_{L}\left|D_{O}(\varphi)\right\rangle=\sigma_{L}\left|D_{O}(\pi-\varphi)\right\rangle=\left|N_{O}(\varphi)\right\rangle, & \sigma_{R}\left|D_{O}(\varphi)\right\rangle=\sigma_{R}\left|D_{O}(\pi-\varphi)\right\rangle=\left|N_{O}\left(\frac{\pi}{2}-\varphi\right)\right\rangle, \\
\sigma_{L}\left|N_{O}(\tilde{\varphi})\right\rangle=\left|D_{O}(\tilde{\varphi})\right\rangle+\left|D_{O}(\pi-\tilde{\varphi})\right\rangle, & \sigma_{R}\left|N_{O}(\tilde{\varphi})\right\rangle=\left|D_{O}\left(\frac{\pi}{2}-\tilde{\varphi}\right)\right\rangle+\left|D_{O}\left(\frac{\pi}{2}+\tilde{\varphi}\right)\right\rangle .
\end{array}
$$

We can write this in terms of defect fusion as

$$
\begin{array}{lll}
X_{\varepsilon} \star D_{O}(\varphi)=D_{O}(\varphi) \star X_{\varepsilon}=D_{O}(\pi-\varphi), & X_{\varepsilon} \star N_{O}(\tilde{\varphi})=N_{O}(\tilde{\varphi}) \star X_{\varepsilon}=N_{O}(\tilde{\varphi}), \\
X_{\sigma} \star D_{O}(\varphi)=N_{O}(\varphi), & D_{O}(\varphi) \star X_{\sigma}=N_{O}\left(\frac{\pi}{2}-\varphi\right), \\
X_{\sigma} \star N_{O}(\tilde{\varphi})=D_{O}(\tilde{\varphi})+D_{O}(\pi-\tilde{\varphi}), & N_{O}(\tilde{\varphi}) \star X_{\sigma}=D_{O}\left(\frac{\pi}{2}-\tilde{\varphi}\right)+D_{O}\left(\frac{\pi}{2}+\tilde{\varphi}\right) .
\end{array}
$$




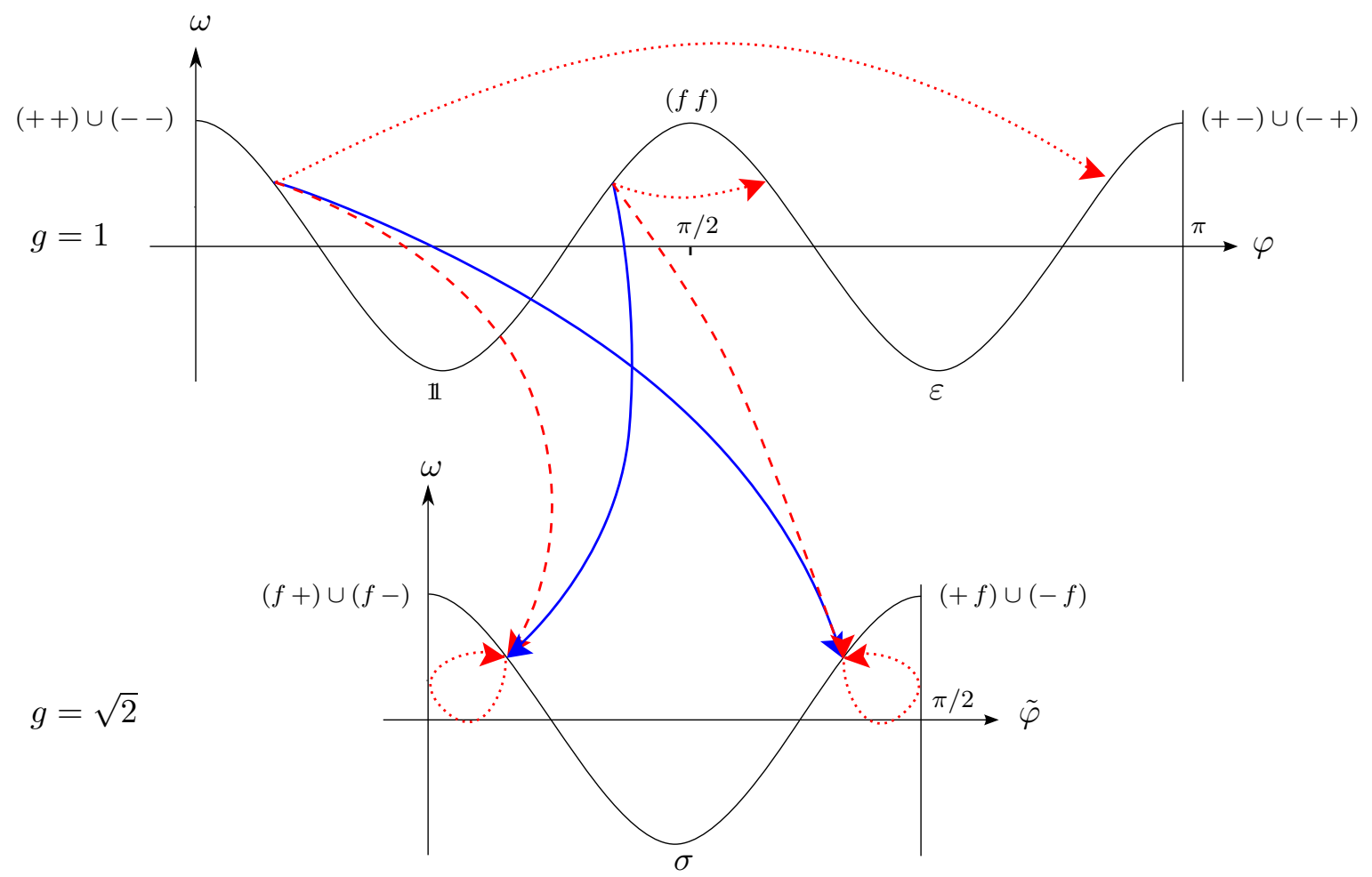

Figure 1: The values of $\omega$ and $g$, and the action of the topological defects on the Dirichlet and Neumann lines of conformal defects in the Ising model. The action of $X_{\varepsilon}$ from the left and from the right is the same and is given by the dotted arrows, the action of $X_{\sigma}$ on the left by the dashed arrows and on the right by the solid arrows. Note that the end points of the Dirichlet and Neumann lines are superpositions of two elementary defects as indicated.

This means that the number $\omega$ does not uniquely specify the orbit under the action of the group-like topological defects, nor do the pair $(\omega, g)$ where $g$ is the ground-state-degeneracy of the boundary condition in the folded model as indicated in figure 1. For a given pair $(\omega, g)$, there are two orbits of the group-like defects. On the other hand, starting from any one elementary defect on the Dirichlet or Neumann line at a given value of $\omega$, all other elementary defects with this $\omega$ can be generated by the action of topological defects (in the sense explained in section 2.2).

In figure 1 we give a pictorial summary of the value of $\omega$ and the actions of $\varepsilon$ and $\sigma$ on the Dirichlet and Neumann line of conformal defects in the Ising model.

Finally we return to the question of extra defects in the orbifold model. Neither the classification of boundary states of [41] nor the explicit calculations of [43, 42] appear to have been performed for the orbifold model, so the comments here remain speculative. The line of exceptional boundary states described in 44, 43, 42, are invariant under the orbifold action $\varphi \mapsto-\varphi$ so we might expect that they would lead to two sets of defects by the addition of twisted sectors, but since both the exceptional boundary states and all states in the twisted sectors have zero overlap with $|W \bar{W}\rangle$ we would expect them to have $\omega=0$ and hence $\mathcal{R}=\mathcal{T}=1 / 2$. 


\subsection{Free fermions}

As stated in the beginning of this section, by the Ising model we denote the modular invariant theory. Free fermions can then be understood as non-local or disorder fields in the Ising model. Defects for free fermions have been considered before, for example in [33].

The simplest class of conformal defects in the free fermion model are those that satisfy

$$
\begin{aligned}
\psi_{m} \hat{D} & =T^{11} \hat{D} \psi_{m}+T^{12} \bar{\psi}_{-m} \hat{D}, \\
\hat{D} \bar{\psi}_{-m} & =T^{21} \hat{D} \psi_{m}+T^{22} \bar{\psi}_{-m} \hat{D} .
\end{aligned}
$$

Compatibility with the fermion algebra implies that the matrix $T$ takes the form

$$
T=\left(\begin{array}{cc}
\cos (\chi) & i \sin (\chi) \\
i \eta \sin (\chi) & \eta \cos (\chi)
\end{array}\right)
$$

where $\eta= \pm 1$. Reference [33] deals primarily with massive fermions in the defect picture but it is easy to take the massless limit and they find that the defects treated there all satisfy (4.17) and (4.18) with $\eta=1$.

The defects in the local Ising model are not expected to be in one-to-one correspondence with those in the free fermion model, but they are related as the Ising model contains certain combinations of the free fermion fields, notably the primary field

$$
\varepsilon=i \bar{\psi} \psi
$$

This enables one to determine the following combinations:

$$
\begin{aligned}
& T^{12}=\left\langle 0\left|\bar{\psi}_{1 / 2} \psi_{1 / 2} \hat{D}\right| 0\right\rangle /\langle 0|\hat{D}| 0\rangle=-i\langle\varepsilon|\hat{D}| 0\rangle /\langle 0|\hat{D}| 0\rangle \\
& T^{21}=\left\langle 0\left|\hat{D} \bar{\psi}_{-1 / 2} \psi_{-1 / 2}\right| 0\right\rangle /\langle 0|\hat{D}| 0\rangle=-i\langle 0|\hat{D}| \varepsilon\rangle /\langle 0|\hat{D}| 0\rangle \\
& T^{12} T^{21}-T^{11} T^{22}=\left\langle 0\left|\bar{\psi}_{1 / 2} \psi_{1 / 2} \hat{D} \bar{\psi}_{-1 / 2} \psi_{-1 / 2}\right| 0\right\rangle /\langle 0|\hat{D}| 0\rangle=-\langle\varepsilon|\hat{D}| \varepsilon\rangle /\langle 0|\hat{D}| 0\rangle .
\end{aligned}
$$

To calculate these using the boundary state formalism we need to identify the fields $\varepsilon^{1}, \varepsilon^{2}$ in the doubled model corresponding to the field $\varepsilon$ on the left and on the right of the defect. The fields $\varepsilon^{i}$ both have conformal dimensions $\left(\frac{1}{2}, \frac{1}{2}\right)$ and there are two primary fields with these weights in the orbifolded free boson. If we use the convention of [33] that the reflection factor for fermion modes at a 'fixed' boundary is $-i$ and at a 'free' boundary is $i$ then we find agreement with our assignment of factorising boundary conditions with the choice

$$
\begin{aligned}
& \left|\varepsilon^{1}\right\rangle=\frac{1}{2}(|(0,2)\rangle+|(0,-2)\rangle-|(1,0)\rangle-|(-1,0)\rangle), \\
& \left|\varepsilon^{2}\right\rangle=\frac{1}{2}(|(0,2)\rangle+|(0,-2)\rangle+|(1,0)\rangle+|(-1,0)\rangle) \\
& \left|\varepsilon^{1} \varepsilon^{2}\right\rangle=a_{-1} \bar{a}_{-1}|0\rangle
\end{aligned}
$$

where $|(m, n)\rangle$ are the free boson vacuum states described earlier and $a_{-1}$ and $\bar{a}_{-1}$ are modes of the free boson field. 
With these choices, one finds for the Neumann defects $N_{O}\left(\tilde{\varphi}_{0}\right)$ that

$$
T^{11}=-T^{22}=i \cos \left(2 \tilde{\varphi}_{0}\right)
$$

so that these cannot be identified with any of the defects in [33 for any value of $\chi$ since they all have $T^{11}=T^{22}$.

For the Dirichlet defects $D_{O}\left(\varphi_{0}\right)$, however,

$$
T^{12}=T^{21}=-i \cos \left(2 \varphi_{0}\right), T^{11} T^{22}=\sin ^{2}\left(2 \varphi_{0}\right),
$$

so that $\eta=1$ in (4.18), and hence also $T^{11}=T^{22}$. These defects can thus be identified with the defects in [33] with

$$
\varphi_{0}= \pm \frac{1}{2}(\chi+\pi / 2) .
$$

The ambiguity in $\chi$ corresponds to the ambiguity in the definition of the fermion fields. As a final comment we note that our quantities $\mathcal{R}$ and $\mathcal{T}$ can again be identified as the reflection and transmission probabilities for the modes of the free fermion.

\section{Conformal defects from coset decompositions}

Consider two conformal field theories $\mathrm{CFT}_{1}$ and $\mathrm{CFT}_{2}$ which are joined along a defect, and denote their chiral algebras by $\mathcal{A}_{1}$ and $\mathcal{A}_{2}$, respectively. For each embedding of another chiral algebra $\mathcal{B}$ into the symmetry algebra $\mathcal{A}_{1} \otimes \mathcal{A}_{2}$ of the product theory $\mathrm{CFT}_{1} \otimes \overline{\mathrm{CFT}}_{2}$ one can then construct a family of non-trivial conformal defects in the following way. First, we analyse the original theory with respect to the reduced symmetry

$$
\mathcal{A}_{1} \otimes \mathcal{A}_{2} \rightarrow \mathcal{B} \otimes\left(\mathcal{A}_{1} \otimes \mathcal{A}_{2}\right) / \mathcal{B}
$$

where the coset chiral algebra $\left(\mathcal{A}_{1} \otimes \mathcal{A}_{2}\right) / \mathcal{B}$ contains the fields which commute with all the operators in $\mathcal{B} .^{5}$ On the practical level reducing the symmetry means that we have to decompose the Hilbert space of the folded model in terms of representations of the smaller symmetry. The theory itself obviously does not change.

In a second step we impose boundary conditions which just preserve the chiral algebra on the right hand side of (5.1). This procedure defines defects between $\mathrm{CFT}_{1}$ and $\mathrm{CFT}_{2}$, which in general are neither fully reflective nor fully transmissive [13]. Note that the interpretation of which excitations are transmitted and which reflected is somewhat mysterious since the coupling of the two theories takes place in the coset part. There is, however, one special class of defects where the physical interpretation is immediately obvious and a concrete formula for the transmission can easily be derived. This will be the subject of the following subsection. Later we will return to the general case and discuss one example in detail.

\footnotetext{
${ }^{5}$ The interpretation of this decomposition is most straightforward if the theories on the two sides of the defect are WZW models based on some groups $G_{1}$ and $G_{2}$. In that case one can define the chiral subalgebra $\mathcal{B}$ in terms of a WZW model related to a common subgroup of $G_{1}$ and $G_{2}$. The coset part on the other hand is described by the standard GKO construction.
} 


\subsection{Transmission of a common sub-symmetry}

One of the most intuitive examples of semi-permeable defect lines arises if the conformal field theories on the two sides of the defect share a common chiral subalgebra $\mathcal{C}$. In that case we can employ the previous construction with $\mathcal{B}=\mathcal{C} \otimes \mathcal{C}$ such that the first factor $\mathcal{C}$ is embedded into $\mathcal{A}_{1}$ and the second into $\mathcal{A}_{2}$. It is then straightforward to construct conformal defects which allow all excitations with respect to the sub-symmetry $\mathcal{C}$ to pass through unaffectedly. In the folded picture this corresponds to boundary conditions which just preserve the sub-symmetry

$$
\mathcal{A}_{1} \otimes \mathcal{A}_{2} \rightarrow \mathcal{A}_{1} / \mathcal{C} \otimes \mathcal{A}_{2} / \mathcal{C} \otimes \mathcal{C} \otimes \mathcal{C}
$$

of the full bulk system and where one has permutation type boundary conditions in the product of the two $\mathcal{C}$-sectors. In the two coset sectors on the other hand we assume trivial gluing conditions.

In order to determine the transmission we need to calculate the correlation functions between the fields $T^{1}=T^{\mathcal{A}_{1} / \mathcal{C}}+T^{1, \mathcal{C}}$ and $T^{2}=T^{\mathcal{A}_{2} / \mathcal{C}}+T^{2, \mathcal{C}}$ as well as their antiholomorphic counterparts in the presence of the boundary. On the level of the individual energy momentum tensors our choice of boundary condition corresponds to the gluing conditions

$$
T^{\mathcal{A}_{1} / \mathcal{C}}=\bar{T}^{\mathcal{A}_{1} / \mathcal{C}} \quad T^{\mathcal{A}_{2} / \mathcal{C}}=\bar{T}^{\mathcal{A}_{2} / \mathcal{C}} \quad T^{1, \mathcal{C}}=\bar{T}^{2, \mathcal{C}} \quad T^{2, \mathcal{C}}=\bar{T}^{1, \mathcal{C}}
$$

on the real axis. These allow us to simplify the boundary correlation functions considerably. For instance we obtain

$$
\begin{aligned}
\left\langle 0\left|L_{2}^{1} \bar{L}_{2}^{2}\right| b\right\rangle & =\left\langle 0\left|\left(L_{2}^{\mathcal{A}_{1} / \mathcal{C}}+L_{2}^{1, \mathcal{C}}\right)\left(\bar{L}_{2}^{\mathcal{A}_{2} / \mathcal{C}}+\bar{L}_{2}^{2, \mathcal{C}}\right)\right| b\right\rangle \\
& =\left\langle 0\left|\left(L_{2}^{\mathcal{A}_{1} / \mathcal{C}}+L_{2}^{1, \mathcal{C}}\right)\left(L_{-2}^{\mathcal{A}_{2} / \mathcal{C}}+L_{-2}^{1, \mathcal{C}}\right)\right| b\right\rangle=\frac{1}{2} c\langle 0 \mid b\rangle .
\end{aligned}
$$

Here, $c$ denotes the Virasoro central charges of $\mathcal{C}$ and we will similarly use the symbols $c_{1,2}$ for the central charges of the chiral algebras $\mathcal{A}_{1,2}$. It is then a simple exercise to plug these expressions into the defining formula (2.9) for the transmission, yielding

$$
\mathcal{T}=\frac{2 c}{c_{1}+c_{2}}
$$

for this specific type of defect. In accordance with physical intuition the quantity (5.5) takes values between 0 and 1 , at least for unitary theories. Moreover, it is maximised if the chiral algebra of one of the models is contained in the one of the other. We would like to stress that in our derivation no reference has been made to the concrete form of the boundary states or similar data. The reader who is interested in these objects should consult the references [13, 20] for further details of the construction.

In order to gain some intuition let us consider a defect between two WZW models based on the group $S U(2)$ and with levels $k_{1}$ and $k_{2}$, respectively. Both WZW models share a common sub-symmetry $U(1) .{ }^{6}$ With the concrete expressions for the central charges we

\footnotetext{
${ }^{6}$ In order to conform to the condition that the chiral subalgebras agree we have to choose the unextended Heisenberg algebra $\mathcal{C}=\hat{u}(1)$ as the common symmetry.
} 
immediately find

$$
\mathcal{T}\left(k_{1}, k_{2}\right)=\frac{\left(k_{1}+2\right)\left(k_{2}+2\right)}{3\left(k_{1} k_{2}+k_{1}+k_{2}\right)} .
$$

It may be checked that the transmission takes values in the interval between $1 / 3$ and 1 . Full transmission $\mathcal{T}=1$ is only achieved when $k_{1}=k_{2}=1$. This is not surprising since in this case one has the equivalence $S U(2)_{1}=U(1)_{1}$, rendering the cosets trivial. The other limiting case $\mathcal{T}=1 / 3$ is obtained in the regime of large levels. Let us finally state the result $\mathcal{T}=(k+2) / 3 k$ which arises if both levels are chosen equal to $k$. A diagram of the transmission coefficients can be found in figure 2 a) below.

Defects of the type considered in this subsection also exist if the WZW models on the two sides are based on different groups, or for cosets. We list some examples in table 1 .

\subsection{An example for the general case}

After the exhaustive discussion of the special case $\mathcal{B}=\mathcal{C} \otimes \mathcal{C}$ we would like to return to a general defect involving a symmetry breaking of the form (5.1). If the subalgebra $\mathcal{B}$ is not of the above factorised from, and hence the embedding of $\mathcal{B}$ into $\mathcal{A}_{1} \otimes \mathcal{A}_{2}$ is not a product of two separate embeddings as in the previous section, a physical interpretation regarding which degrees of freedom are transmitted and which reflected is not obvious. Having access to the value of the transmission is then a good hint about the actual nature of the defect.

The calculation of the transmission for decompositions of the form (5.1) requires rather detailed knowledge about the representation theory of the chiral algebras involved. For simplicity we will thus specifically consider the coset decompositions

$$
S U(2)_{k_{1}} \times S U(2)_{k_{2}} \rightarrow \frac{S U(2)_{k_{1}} \times S U(2)_{k_{2}}}{S U(2)_{k_{1}+k_{2}}} \times S U(2)_{k_{1}+k_{2}}
$$

in order to explain the main steps. These examples also have the advantage of making contact to the previous subsection where a different type of defect between the same conformal field theories has been discussed. But in contrast to the last case there is no general

\begin{tabular}{cccc}
$\mathrm{CFT}_{1}$ & $\mathrm{CFT}_{2}$ & Symmetry preserved & Transmission $\mathcal{T}$ \\
\hline$\left(\mathcal{G}_{1}\right)_{k_{1}}$ & $\left(\mathcal{G}_{2}\right)_{k_{2}}$ & $\frac{\mathcal{G}_{1} \times \mathcal{G}_{2}}{U(1)^{l} \times U(1)^{l}} \times U(1)^{l} \times U(1)^{l}$ & $\frac{2 l\left(k_{1}+g_{1}^{\vee}\right)\left(k_{2}+g_{2}^{\vee}\right)}{k_{1}\left(k_{2}+g_{2}^{\vee}\right) \operatorname{dim} \mathcal{G}_{1}+k_{2}\left(k_{1}+g_{1}^{\vee}\right) \operatorname{dim} \mathcal{G}_{2}}$ \\
$S U(3)_{k}$ & $S U(3)_{4 k}$ & $\frac{S U(3)_{k}}{S U(2)_{4 k}} \times \frac{S U(3)_{4 k}}{S U(2)_{4 k}} \times S U(2)_{4 k} \times S U(2)_{4 k}$ & $\frac{3(k+3)(4 k+3)}{2(2 k+1)(8 k+15)}$ \\
$\left(F_{4}\right)_{k}$ & $\left(E_{6}\right)_{k}$ & $\frac{\left(F_{4}\right)_{k}}{\left(G_{2}\right)_{k}} \times \frac{\left(E_{6}\right)_{k}}{\left(G_{2}\right)_{k}} \times\left(G_{2}\right)_{k} \times\left(G_{2}\right)_{k}$ & $\frac{14(k+9)(k+12)}{13(k+4)(5 k+51)}$ \\
$S U(2)_{k}$ & $\frac{S U(2)_{k}}{U(1)_{k}}$ & $\frac{S U(2)}{U(1)} \times \frac{S U(2)}{U(1)} \times U(1)$ & $\frac{4(k-1)}{5 k-2}$ \\
$S U(2)_{k_{1}}$ & $S U(2)_{k_{2}}$ & $\frac{S U(2)_{k_{1}} \times S U(2)_{k_{2}}}{S U(2)_{k_{1}+k_{2}}} \times S U(2)_{k_{1}+k_{2}}$ & $\frac{4 k_{1} k_{2}\left(k_{1}+2\right)\left(k_{2}+2\right)}{\left(k_{1} k_{2}+k_{1}+k_{2}\right)\left(k_{1}+k_{2}\right)\left(k_{1}+k_{2}+4\right)} \frac{1+\cos \frac{2 \pi}{k_{1}+k_{2}+2}}{1+2 \cos \frac{2 \pi}{k_{1}+k_{2}+2}}$
\end{tabular}

Table 1: List of some defect systems and their respective transmissions. The first four examples are of the form treated in section 5.1. The parameter $l$ in the first line obeys $1 \leq l \leq$ $\min \left(\operatorname{rank} \mathcal{G}_{1}, \operatorname{rank} \mathcal{G}_{2}\right)$. In the last line (see section 5.2) $k_{1}, k_{2}$ are odd, and only the maximal value for $\mathcal{T}$ is listed. 
and simple derivation of the transmission amplitude available in this case. Instead, we are forced into a relatively voluminous and model specific calculation starting from the definition (2.5). The relevant boundary states $|B\rangle$ are given in terms of Ishibashi states which preserve the right hand side of (5.7) and which are themselves sums over tensor products of a set of orthonormalised states in irreducible representations of the reduced symmetry. Consequently, we need to express the states

$$
L_{-2}^{i} \bar{L}_{-2}^{j}|0\rangle
$$

in terms of an orthonormal basis of the corresponding representation spaces.

The states we are interested in reside in the vacuum module of $S U(2)_{k_{1}} \times S U(2)_{k_{2}}$. In order to identify these states after the coset decomposition (5.7) we have to work out the branching functions of the affine representations involved. Since the states (5.8) have $h=2$ and are all singlets with respect to the zero mode algebra of $S U(2)_{k_{12}}$, where $k_{12}=k_{1}+k_{2}$, we can restrict our attention to the first few characters $\chi_{0}^{\left(k_{12}\right)}, \chi_{1}^{\left(k_{12}\right)}$ and $\chi_{2}^{\left(k_{12}\right)}$ of the diagonal affine subalgebra $S U(2)_{k_{12}}$. When writing down the characters it is useful to keep track of the representation content with respect to the $s u(2)$ zero mode algebra on each energy level. For $k \geq 2$ the relevant characters of $S U(2)_{k}$ can then easily be found to be (up to higher orders in $q$ and not spelling out the overall prefactor $q^{h-c / 24}$ )

$$
\begin{aligned}
& \chi_{0}^{(k)}(q)=(0)+q(1)+q^{2}((0)+(1)+(2))+\cdots \\
& \chi_{1}^{(k)}(q)=(1)+q((0)+(1)+(2))+\cdots \\
& \chi_{2}^{(k)}(q)=(2)+q((1)+(2)+(3))+\cdots .
\end{aligned}
$$

Here, the symbols $(j)$ denote an $s u(2)$ representation of spin $j$. For a first hint towards where to search for the relevant states (5.8) in the decomposed theory it is useful to start with the following equality, which involves the affine characters of $S U(2)$ and is valid for $k_{1}, k_{2} \geq 2$,

$$
\begin{aligned}
\chi_{0}^{\left(k_{1}\right)}(q) \chi_{0}^{\left(k_{2}\right)}(q)= & (0)+2 q(1)+3 q^{2}((0)+(1)+(2))+\cdots \\
= & \chi_{0}^{\left(k_{12}\right)}(q)\left(1+q^{2}+\cdots\right)+\chi_{1}^{\left(k_{12}\right)}(q) q(1+q+\cdots) \\
& +\chi_{2}^{\left(k_{12}\right)}(q) q^{2}(1+\cdots)+\cdots
\end{aligned}
$$

where in the first line we decomposed the tensor product of the two vacuum modules with respect to the zero mode action of the diagonal $S U(2)_{k_{12}}$. From (5.9) we see that the representations (0) and (1) of $S U(2)_{k_{12}}$ contain singlets at levels 0 and 2, and 1, respectively, while (2) does not have a singlet at level 0 . The three level 2 singlets in $(5.10)$ thus lie in $(0,0,0) \times(0)$ and $(0,0,1) \times(1)$. In this notation, the triple refers to a representation of the diagonal $S U(2)$ coset while the last label specifies a representation of $S U(2)_{k_{12}}$. In particular, the desired states (5.8) are a linear combination of maximally three states inside of $(0,0,0) \times(0)$ and $(0,0,1) \times(1)$. 
With a bit of work one finds that the primary states corresponding to the representations just mentioned are given by $|(0,0,0)\rangle \otimes|0\rangle=|0\rangle \otimes|0\rangle$ as well as ${ }^{7}$

$$
|(0,0,1)\rangle \otimes|1\rangle=\frac{1}{\sqrt{k_{1} k_{2}\left(k_{1}+k_{2}\right)}}\left(k_{2} E_{-1}^{+}|0\rangle \otimes|0\rangle-k_{1}|0\rangle \otimes E_{-1}^{+}|0\rangle\right) .
$$

Here, the left hand side refers to the coset decomposition while the right hand side is the corresponding expression in the product of affine representations of $S U(2)_{k_{1}}$ and $S U(2)_{k_{2}}$. By acting with creation operators on the two states above one reveals the orthonormal singlet states $\left|v_{i}\right\rangle, i=1,2,3$, we are interested in. The first two of these lie in $(0,0,0) \times(0)$ and correspond via the state field correspondence to the energy momentum tensors of the coset theory and the affine subalgebra, respectively. The third state belongs to a descendent of the affine highest weight state $|1\rangle$. Explicit formulas for the vectors $v_{i}$ can be found in the appendix in eq. (B.3).

Using the vectors $v_{i}$, the states (5.8) corresponding to the energy momentum tensors of the individual theories $S U(2)_{k_{1}}$ and $S U(2)_{k_{2}}$ can be expressed in an adapted basis, see eqs. (B.4) and (B.5). In order to calculate the transmission we then have to evaluate all the overlaps

$$
\left\langle 0\left|L_{2}^{i} \bar{L}_{2}^{j}\right| b\right\rangle \text {. }
$$

For the present choice of gluing conditions the boundary states are labelled by a triple $\left(\rho_{1}, \rho_{2}, \rho\right)$ whose entries label representations of the three affine algebras involved [13], i.e. they satisfy $2 \rho_{i} \in\left\{0,1, \cdots, k_{i}\right\}$ and $2 \rho \in\left\{0,1, \cdots, k_{1}+k_{2}\right\}$. In addition there is an identification $\left(\rho_{1}, \rho_{2}, \rho\right) \sim\left(k_{1} / 2-\rho_{1}, k_{2} / 2-\rho_{2},\left(k_{1}+k_{2}\right) / 2-\rho\right)$ (but no selection rule).

For the actual calculation of the overlap (5.12) we need to expand the boundary state in terms of Ishibashi states which implement the proper gluing conditions. The corresponding expressions are 13.

$$
\left.\left.\left|\left(\rho_{1}, \rho_{2}, \rho\right)\right\rangle=\sum_{\substack{\mu_{1}, \mu_{2}, \mu \\ \mu_{1}+\mu_{2}+\mu \in \mathbb{Z}}} \frac{S_{\rho_{1} \mu_{1}}^{\left(k_{1}\right)} S_{\rho_{2} \mu_{2}}^{\left(k_{2}\right)}}{\sqrt{S_{0 \mu_{1}}^{\left(k_{1}\right)} S_{0 \mu_{2}}^{\left(k_{2}\right)}}} \frac{S_{\rho \mu}^{\left(k_{1}+k_{2}\right)}}{S_{0 \mu}^{\left(k_{1}+k_{2}\right)}}\left|\left(\mu_{1}, \mu_{2}, \mu\right)\right\rangle\right\rangle \otimes|\mu\rangle\right\rangle .
$$

The sum runs over the ranges $2 \mu_{i} \in\left\{0,1, \cdots, k_{i}\right\}$ and $2 \mu \in\left\{0,1, \cdots, k_{1}+k_{2}\right\}$ and involves elements of the modular S matrices of $S U(2)_{k}$ which are given by

$$
S_{j_{1} j_{2}}^{(k)}=\sqrt{\frac{2}{k+2}} \sin \frac{\pi}{k+2}\left(2 j_{1}+1\right)\left(2 j_{2}+1\right) .
$$

We will assume that at least one of the levels is odd in order to avoid problems with fixed point resolution. (If all levels are even, one needs to consider the boundary state $\left|\left(k_{1} / 4, k_{2} / 4,\left(k_{1}+k_{2}\right) / 4\right)\right\rangle$ which is the sum of two elementary ones. $)$ It is worth emphasising that among the previous boundary states one can recover Cardy states by considering boundary labels of the form $\left(\rho_{1}, \rho_{2}, 0\right)$. Since these correspond to factorising boundary conditions the transmission has to vanish in this case and indeed that is what will be confirmed by our general formula below.

\footnotetext{
${ }^{7}$ Our current algebra conventions are summarised in eq. (B.2.).
} 
In the overlap (5.12) we are interested in, only two sectors of the boundary state (5.13) actually contribute, namely the ones with $\mu_{1}=\mu_{2}=0$ and $\mu \in\{0,1\}$. The Ishibashi states are a sum over a complete set of orthonormal states. Hence the relevant contribution in the present case is

$\left|\left(\rho_{1}, \rho_{2}, \rho\right)\right\rangle=\mathcal{N}\left(\rho_{1}, \rho_{2}, \rho\right)\left[\left(\left|v_{1}\right\rangle \otimes \overline{\left|v_{1}\right\rangle}+\left|v_{2}\right\rangle \otimes \overline{\left|v_{2}\right\rangle}\right)+\frac{S_{00}^{\left(k_{1}+k_{2}\right)} S_{\rho 1}^{\left(k_{1}+k_{2}\right)}}{S_{\rho 0}^{\left(k_{1}+k_{2}\right)} S_{01}^{\left(k_{1}+k_{2}\right)}}\left|v_{3}\right\rangle \otimes \overline{\left|v_{3}\right\rangle}\right]+\cdots$

with a normalisation $\mathcal{N}\left(\rho_{1}, \rho_{2}, \rho\right)$ which will drop out in the final expression for the transmission. After a straightforward calculation which uses the values $c_{k}=3 k /(k+2)$ of the central charges and the states defined in eq. (B.5) we finally arrive at a nice expression for the transmission, which turns out to be independent of $\rho_{1}$ and $\rho_{2}$,

$$
\mathcal{T}_{\rho}\left(k_{1}, k_{2}\right)=\frac{2 k_{1} k_{2}\left(k_{1}+2\right)\left(k_{2}+2\right)}{\left(k_{1} k_{2}+k_{1}+k_{2}\right)\left(k_{1}+k_{2}\right)\left(k_{1}+k_{2}+4\right)}\left\{1-\frac{S_{\rho 1}^{\left(k_{1}+k_{2}\right)} S_{00}^{\left(k_{1}+k_{2}\right)}}{S_{\rho 0}^{\left(k_{1}+k_{2}\right)} S_{01}^{\left(k_{1}+k_{2}\right)}}\right\} .
$$

It is then a natural problem to investigate the level dependence of the labels $\rho_{\min }$ and $\rho_{\max }$ which minimise or maximise the transmission, respectively. To do so we have to extremise the quotient

$$
q(\rho)=-\frac{S_{\rho 1}^{\left(k_{1}+k_{2}\right)} S_{00}^{\left(k_{1}+k_{2}\right)}}{S_{\rho 0}^{\left(k_{1}+k_{2}\right)} S_{01}^{\left(k_{1}+k_{2}\right)}}=-\frac{\sin \frac{3 \pi(2 \rho+1)}{k_{1}+k_{2}+2}}{\sin \frac{3 \pi}{k_{1}+k_{2}+2}} \frac{\sin \frac{\pi}{k_{1}+k_{2}+2}}{\sin \frac{\pi(2 \rho+1)}{k_{1}+k_{2}+2}}=-\frac{1+2 \cos \frac{2 \pi(2 \rho+1)}{k_{1}+k_{2}+2}}{1+2 \cos \frac{2 \pi}{k_{1}+k_{2}+2}} .
$$

In the relevant interval for $\rho$ this function has one maximum and two minima, namely

$$
\begin{aligned}
& \rho_{\max }=\frac{k_{1}+k_{2}}{4} \quad q\left(\rho_{\max }\right)=\frac{1}{1+2 \cos \frac{2 \pi}{k_{1}+k_{2}+2}} \stackrel{k_{1}+k_{2} \rightarrow \infty}{\longrightarrow} \frac{1}{3} \\
& \rho_{\min } \in\left\{0, \frac{k_{1}+k_{2}}{2}\right\} q\left(\rho_{\min }\right)=-1 .
\end{aligned}
$$

It should be noted that the actual maximum of the function $q(\rho)$ is not realised in the range of valid boundary labels if the sum of the levels is odd. In that case the maximal transmission is found for $\rho=\rho_{\max } \pm 1 / 4$.

Let us now discuss whether the transmission we calculated satisfies all the relevant consistency conditions. First of all, the previous calculation provides an explicit proof that the minimal transmission for the considered type of defect is indeed zero: $\mathcal{T}_{\min }=\mathcal{T}_{\rho_{\min }}=0$. We notice that vanishing transmission is realised precisely for $\rho=0$ (the second value is related to 0 by the identification rule) where the boundary state (5.13) reduces to the product of two Cardy states of $S U(2)_{k_{1}}$ and $S U(2)_{k_{2}}$, respectively. On the other hand the maximal transmission is given by

$$
\mathcal{T}_{\max }\left(k_{1}, k_{2}\right)=\frac{4 k_{1} k_{2}\left(k_{1}+2\right)\left(k_{2}+2\right)}{\left(k_{1} k_{2}+k_{1}+k_{2}\right)\left(k_{1}+k_{2}\right)\left(k_{1}+k_{2}+4\right)}\left\{\begin{array}{l}
\frac{1+\cos \frac{2 \pi}{k_{1}+k_{2}+2}}{1+2 \cos \frac{2 \pi}{k_{1}+k_{2}+2}} \text { for } k_{1}, k_{2} \text { odd } \\
\frac{1+\cos \frac{\pi}{k_{1}+k_{2}+2}}{1+2 \cos \frac{\pi}{k_{1}+k_{2}+2}} \text { for } k_{1}+k_{2} \text { odd . }
\end{array}\right.
$$


It is not entirely obvious from this expression but the transmission turns out to be bounded from above by 1 as expected, see diagram $2 \mathrm{~b}$ ). Note that one indeed reaches full transmission for $\rho=1 / 2$ and $k=1$. In fact this could be expected since these states have already been identified in [13] as permutation boundary states of $S U(2)_{1} \times S U(2)_{1}$.

The analysis of (5.19) simplifies considerably in the limit of large levels. In that case one can easily derive the limiting value

$$
\mathcal{T}_{\max }\left(k_{1}, k_{2}\right) \stackrel{k_{1}, k_{2} \gg 1}{\longrightarrow} \frac{8 k_{1} k_{2}}{3\left(k_{1}+k_{2}\right)^{2}}
$$

for the maximal transmission for the considered type of defect. Note that this value is equal to $2 / 3$ for $k_{1}=k_{2}$ and smaller otherwise. In particular it is a monotone decreasing function in each of the levels and vanishes when one level is sent to infinity while the other one is kept fixed.

The setting considered here allows us to make contact with the generalised permutation branes which have been discussed in 21 . ${ }^{8}$ Although neither were concrete expressions for the boundary states derived nor could the precise symmetry preserved be worked out, it was argued that, on geometrical grounds, the diagonal current algebra should be preserved. If in the present setup we choose one of the levels to be one, e.g. $k_{2}=1$, then we preserve the diagonal current algebra and, moreover, the additional coset is a Virasoro minimal model. Hence in that case we are able to describe all boundary conditions which preserve the diagonal current algebra and, in particular, all generalised permutation branes. The maximal transmission in that case is easily worked out from eq. (5.19). It would be interesting to be able to compare this result with a calculation genuinely done in the context of generalised permutation branes but up to now their algebraic construction seems to be

${ }^{8}$ We would like to thank Stefan Fredenhagen for pointing this out.

a)

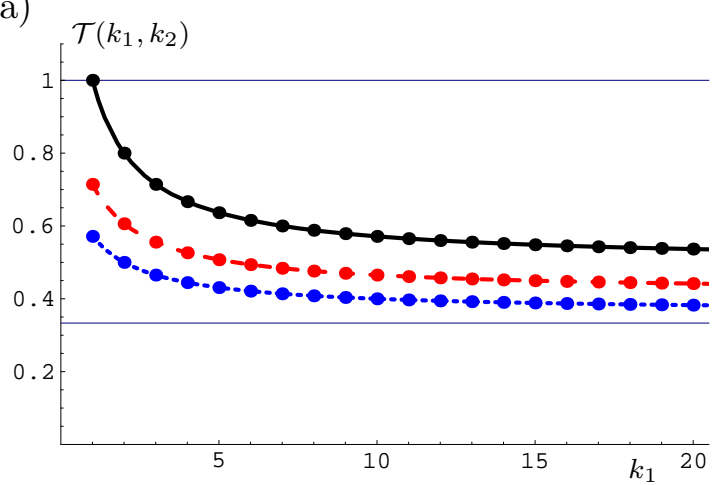

b)

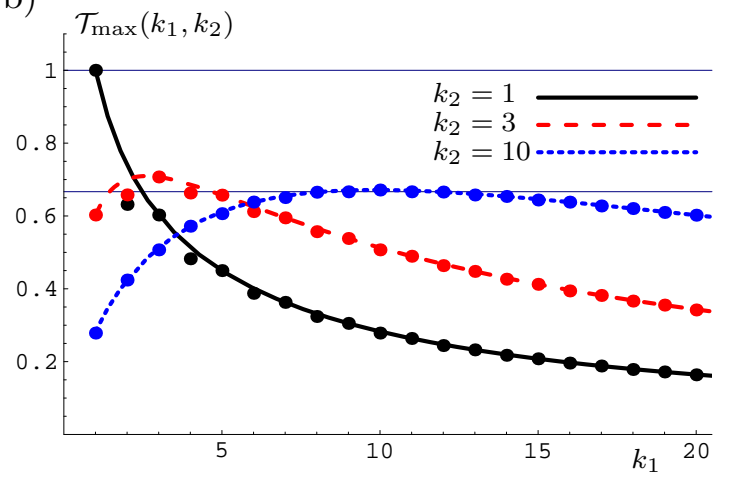

Figure 2: Transmission for specific defects joining $S U(2)_{k_{1}}$ and $S U(2)_{k_{2}}$ in dependence on $k_{1}$ for the choices $k_{2}=1,3,10$ : a) Defect obtained from the common $U(1)$ sub-symmetry (see section 5.1). b) Defect obtained by embedding $S U(2)_{k_{1}+k_{2}}$ (see section 5.2). Here the defect with maximal $\mathcal{T}$ is considered; the line gives $\mathcal{T}_{\rho}$ for $\rho=\frac{1}{4}\left(k_{1}+k_{2}\right)$. For $k_{1}+k_{2}$ even this value is achieved, while for $k_{1}+k_{2}$ odd the maximal $\mathcal{T}$ occurs for $\rho=\frac{1}{4}\left(k_{1}+k_{2} \pm 1\right)$, so that the corresponding points are slightly below the line. 
beyond reach although recent progress in that direction has been reported for products of supersymmetric $S U(2)$ cosets [22].

Before we conclude this section let us briefly investigate the action of the topological defects on the boundary conditions above. In the $S U(2)_{k}$ WZW model the elementary topological defects are labelled by irreducible unitary representations of the affine Lie algebra. In our situation we have two different affine algebras on both sides of the defect. Their action on a defect labelled by $\left(\rho_{1}, \rho_{2}, \rho\right)$ may be expressed in terms of the fusion rules of the individual theories as

$$
X_{j_{1}} * D\left(\rho_{1}, \rho_{2}, \rho\right) * X_{j_{2}}=\sum_{\rho_{1}^{\prime}, \rho_{2}^{\prime}}\left(N^{\left(k_{1}\right)}\right)_{j_{1} \rho_{1}}^{\rho_{1}^{\prime}}\left(N^{\left(k_{2}\right)}\right)_{j_{2} \rho_{2}}^{\rho_{2}^{\prime}} D\left(\rho_{1}^{\prime}, \rho_{2}^{\prime}, \rho\right)
$$

where $2 j_{i} \in\left\{0, \ldots, k_{i}\right\}, i=1,2$. The independence of the transmission amplitude on $\rho_{1}$ and $\rho_{2}$ trivially guarantees that the former is invariant under the action of topological defects.

One can verify that the conformal defects $D\left(\rho_{1}, \rho_{2}, \rho\right)$ for a fixed value of $\mathcal{R}$ are precisely those with a fixed value of $\rho$. In particular, all conformal defects (preserving the coset symmetry) with a given value of $\mathcal{R}$ are generated (in the sense of section 2.2) by the action of topological defects on $D(0,0, \rho)$ for an appropriate $\rho$.

\section{Minimal models with rational products}

In this section we consider the case where $\mathrm{CFT}_{1}$ and $\mathrm{CFT}_{2}$ are (Virasoro) minimal models such that $\mathrm{CFT}_{1} \times \overline{\mathrm{CFT}}_{2}$ is again a minimal model. Since the central charges add, this is only possible if at least one of the two is non-unitary. The fact that the product theory is again a minimal model means one can find all conformal boundary conditions, and hence all conformal defects joining $\mathrm{CFT}_{1}$ and $\mathrm{CFT}_{2}$.

There are three products of minimal models which can be analysed in this way, namely Lee-Yang $\times$ Lee-Yang, Lee-Yang $\times$ Ising and Lee-Yang $\times M_{2,7}$. To see that there cannot be more note that a product of two minimal models has a chiral symmetry given by two copies of the Virasoro algebra. Hence to describe it one has to extend the chiral symmetry of the minimal model used to describe the product. The existence of such an extension implies the existence of a block-diagonal modular invariant partition function, and these have been classified 44]. The block diagonal cases are the $D_{\text {even }}$-series for $M_{p, 2(2 m+1)}$, the $E_{6}$ invariant for $M_{p, 12}$, and the $E_{8}$ invariant for $M_{p, 30}$ (up to the symmetry which interchanges $p$ and $q$ in $\left.M_{p, q}\right)$. Of these, only $D_{6}$ of $M_{3,10}, E_{6}$ of $M_{5,12}$ and $E_{8}$ of $M_{7,30}$ involve an extra field of weight two in the extended symmetry algebra, and these are already the three cases quoted above. Furthermore, these three models are also the only cases in which the sum of the effective central charges of two minimal models is equal to the effective central charge of a third.

In the following three sections we list the results found for these three models. The calculations have been done using the category theoretic methods of [14] and have been shifted to appendices C.2 C.4. 


\subsection{Lee-Yang $\times$ Lee-Yang}

The Lee-Yang model is the minimal model $M_{2,5}$ and has central charge $c=-\frac{22}{5}$. The product $M_{2,5} \times M_{2,5}$ is equivalent to the $D_{6}$-invariant of $M_{3,10}$. The Kac table of the $c=-\frac{22}{5}$ Virasoro algebra contains two irreducible highest weight representations, which have conformal weight 0 and $-\frac{1}{5}$. We will denote them by 1 and $\phi$, respectively. The fusion product of $\phi$ with itself is $\phi \cdot \phi=\mathbf{1}+\phi$. It follows from the methods in [24, 9], which are valid in general for models with charge-conjugation modular invariant, that the Lee-Yang model has two conformal boundary conditions $B_{1}$ and $B_{\phi}$, and two topological defects $X_{1}$ and $X_{\phi}$. The fusion of the topological defects amongst themselves, and their fusion with the boundary conditions, agrees with that of the chiral representations, i.e.

$$
X_{\phi} \star X_{\phi}=X_{1}+X_{\phi} \quad \text { and } \quad X_{\phi} \star B_{1}=B_{\phi} \quad, \quad X_{\phi} \star B_{\phi}=B_{1}+B_{\phi} .
$$

The product model on the upper half-plane therefore has at least six conformal boundary conditions, corresponding to the four factorising conformal defects $F_{\mathbf{1 1}}, F_{\mathbf{1} \phi}, F_{\phi \mathbf{1}}, F_{\phi \phi}$ (where the two labels are the boundary condition for the Lee-Yang model on the upper and lower half-plane, respectively) and the two topological defects $X_{1}$ and $X_{\phi}$.

In fact, the conformal boundary conditions of the $D_{6}$-invariant of $M_{3,10}$ can be labelled by the nodes of the $D_{6}$ Dynkin diagram $[45,46]^{9}$, so the six boundary conditions mentioned above are already all there is. The relation between the Lee-Yang and $M_{3,10}$ quantities is computed in appendix C.2 to be:

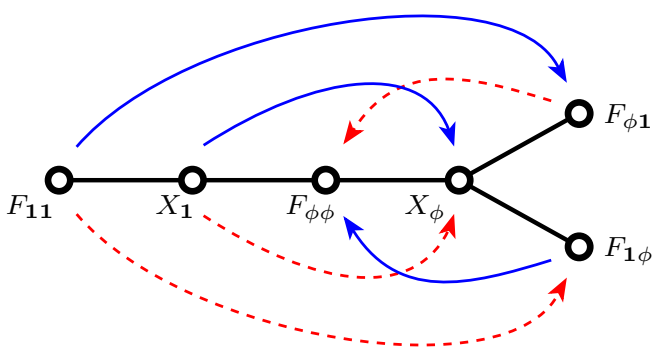

The solid arrows give the result of fusing the corresponding conformal defect with $X_{\phi}$ from the left, and the dashed arrow the result of fusing with $X_{\phi}$ from the right. The arrows not included explicitly in the diagram follow from the rule $X_{\phi} \star X_{\phi}=X_{1}+X_{\phi}$.

Altogether we see that there are six conformally invariant ways to join a Lee-Yang model to itself, and they are either purely reflecting with $\mathcal{R}=1\left(F_{\mathbf{1 1}}, F_{\mathbf{1} \phi}, F_{\phi \mathbf{1}}\right.$ and $\left.F_{\phi \phi}\right)$ or purely transmitting with $\mathcal{T}=1\left(X_{1}\right.$ and $\left.X_{\phi}\right)$.

\subsection{Lee-Yang $\times$ Ising}

The Ising model is the minimal model $M_{3,4}$, and the product $M_{2,5} \times M_{3,4}$ is equivalent to the $E_{6}$-invariant of $M_{5,12}$. The $c=\frac{1}{2}$ Virasoro algebra has three unitary irreducible highest

\footnotetext{
${ }^{9}$ To be more precise, the boundary conditions are labelled by a pair $(x, y)$, where $x$ is a node of the $D_{6}$ Dynkin diagram and $y$ is an odd node of the $A_{2}$ Dynkin diagram. In the present example there is only one choice for $y$ and we have omitted this label, but in the next two examples the $A_{n}$ label appears explicitly.
} 
weight representations. Their conformal weights are $0, \frac{1}{16}$ and $\frac{1}{2}$, and we denote them by $\mathbf{1}$, $\sigma$ and $\varepsilon$, respectively. Their fusion product is given by $\varepsilon \cdot \varepsilon=\mathbf{1}, \sigma \cdot \sigma=1+\varepsilon$ and $\varepsilon \cdot \sigma=\sigma$.

The Ising model has three conformal boundary conditions, which we denote by $B_{\mathbf{1}}$, $B_{\sigma}$ and $B_{\varepsilon}$, as well as three topological defects, labelled $X_{1}, X_{\sigma}$ and $X_{\varepsilon}$. As for the LeeYang model, the fusion of topological defects is given by the fusion of the representations labelling them. For example $X_{\sigma} \star X_{\sigma}=X_{1}+X_{\varepsilon}$ or $X_{\sigma} \star B_{\varepsilon}=B_{\sigma}$.

Consider now the situation where on the upper half-plane we have the Lee-Yang model and on the lower half-plane the Ising model. Since their central charges are different, there cannot be any topological defects. However, there will be six factorising defects, each labelled by a pair of boundary conditions, one for the Lee-Yang model and one for the Ising model: $F_{11}, F_{1 \sigma}, F_{1 \varepsilon}, F_{\phi 1}, F_{\phi \sigma}, F_{\phi \varepsilon}$. The total list of conformal defects is in oneto-one correspondence with conformal boundary conditions of the $E_{6}$-invariant of $M_{5,12}$. There are 12 such boundary conditions, and they are conveniently described by pairs $(x, y)$, with $x$ a node of the $E_{6}$ Dynkin diagram and $y$ an odd node of the $A_{4}$ Dynkin diagram. The calculation in appendix C.3 yields the following result for the action of the topological defects on these 12 conformal defects:

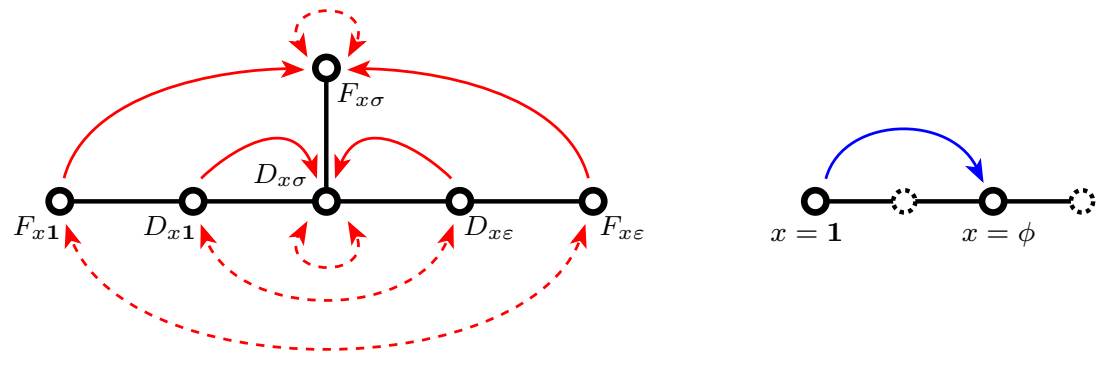

The dashed arrows in the $E_{6}$ diagram give the $\mathbb{Z}_{2}$-action of the $X_{\varepsilon}$ defect, while the solid arrow amounts to the action of $X_{\sigma}$. On the $A_{2}$ diagram, the solid arrow shows the fusion with $X_{\phi}$. Again, the action of the topological defects on the 12 conformal defects can be reconstructed from those shown explicitly in (6.3) together with the fusion product of the topological defects. For example, $X_{\phi} \star F_{1 \varepsilon} \star X_{\varepsilon}=F_{\phi \mathbf{1}}$, or $X_{\phi} \star D_{\phi \sigma} \star X_{\sigma}=D_{1 \mathbf{1}}+D_{\phi \mathbf{1}}+$ $D_{1 \varepsilon}+D_{\phi \varepsilon}$.

For the six factorising defects, $\mathcal{R}\left(F_{x y}\right)=1$, while for the remaining six one finds (see appendix C.3

$$
\mathcal{R}\left(D_{x y}\right)=\frac{2841+440 \sqrt{3}}{1521}=2.3689 . . \quad, \quad \mathcal{T}\left(D_{x y}\right)=1-\mathcal{R}=-1.3689 . . \quad .
$$

This provides an example that $\mathcal{R}$ and $\mathcal{T}$ can lie outside the interval $[0,1]$ when non-unitary models are involved.

\subsection{Lee-Yang $\times \mathrm{M}_{2,7}$}

The product theory $M_{2,5} \times M_{2,7}$ is given by the $E_{8}$-invariant of $M_{7,30}$. The minimal model $M_{2,7}$ has central charge $c=-\frac{68}{7}$, and there are three irreducible Virasoro highest weight representations in the Kac table. These have conformal weights $0,-\frac{2}{7},-\frac{3}{7}$ and will be labelled $1, \alpha, \beta$, respectively. The fusion rules are $\alpha \cdot \alpha=1+\beta, \alpha \cdot \beta=\alpha+\beta$ and 
$\beta \cdot \beta=\mathbf{1}+\alpha+\beta$. There are three conformal boundary conditions $B_{y}$ and three topological defects $X_{y}$ for $M_{2,7}$, with $y$ taking values in $\{\mathbf{1}, \alpha, \beta\}$. The action of the topological defects is again given by the fusion rules of $\alpha$ and $\beta$.

The analysis of the conformal defects which can join $M_{2,5}$ and $M_{2,7}$ is similar to the previous two minimal model examples. There will be six factorising defects and no topological ones. The product model, described as the $E_{8}$-invariant of $M_{7,30}$, has 24 conformal boundary conditions. They are labelled by pairs $(x, y)$, with $x$ a node of the $E_{8}$ Dynkin diagram and $y$ an odd node of the $A_{6}$ Dynkin diagram.

The action of the topological defects of $M_{2,5}$ and $M_{2,7}$ on the conformal defects corresponding to these boundary conditions is given by:
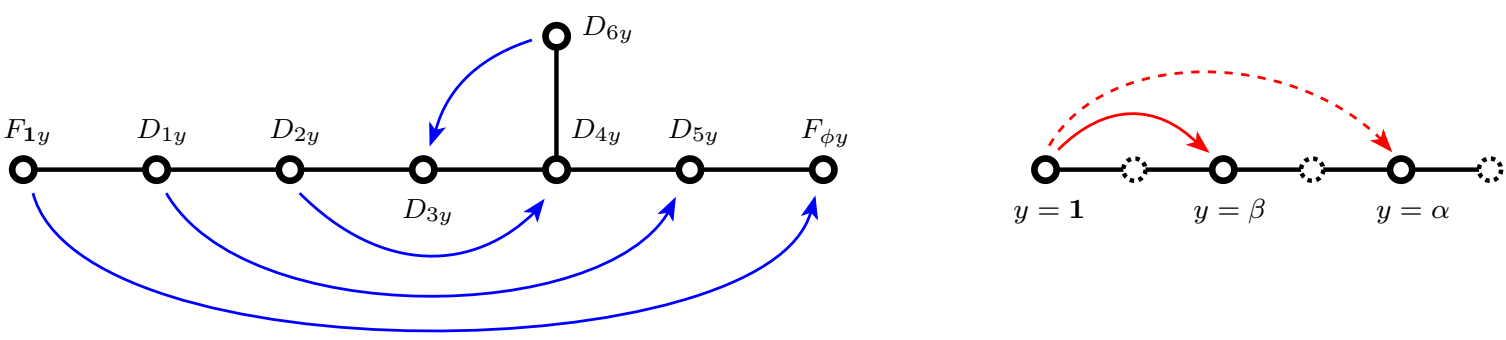

The arrows in the $E_{8}$ picture give the result of fusing with $X_{\phi} \cdot{ }^{10}$ The dashed arrow in the $A_{6}$ diagram gives the fusion with $X_{\alpha}$ and the solid arrow the fusion with $X_{\beta}$.

The exact reflection and transmission coefficients are somewhat cumbersome to spell out because of the fractions arising from the central charges, and it is much briefer to give the coefficients $\omega$ instead, which are related to $\mathcal{R}$ and $\mathcal{T}$ via (2.9). The computations can be found in appendix C.4, and the results are, for $y \in\{\mathbf{1}, \alpha, \beta\}$,

$$
\begin{array}{ll}
\omega\left(F_{1 y}\right)=\omega\left(F_{\phi y}\right)=1 & \Rightarrow \mathcal{R}=1 \\
\omega\left(D_{1 y}\right)=\omega\left(D_{5 y}\right)=\cos \left(\pi \frac{17}{30}\right) / \cos \left(\pi \frac{7}{30}\right) & \Rightarrow \mathcal{R}=0.4508 . . \\
\omega\left(D_{2 y}\right)=\omega\left(D_{4 y}\right)=-\sin \left(\pi \frac{7}{30}\right) / \sin \left(\pi \frac{17}{30}\right) & \Rightarrow \mathcal{R}=0.2773 . . \\
\omega\left(D_{3 y}\right)=\omega\left(D_{6 y}\right)=\cos \left(\pi \frac{1}{30}\right) / \cos \left(\pi \frac{11}{30}\right) \quad \Rightarrow \quad \mathcal{R}=1.6201 . . .
\end{array}
$$

We see that also in this example there are six conformal defects where $\mathcal{R}$ (and hence also $\mathcal{T}$ ) lies outside the interval $[0,1]$.

\section{Conclusions and outlook}

In this article we proposed two new quantities $\mathcal{R}$ and $\mathcal{T}$ which may be used to characterise conformal defects, and we determined their value for a variety of critical defect systems. In the case of the free boson and the free fermion they reduce to the reflection and transmission probability, respectively. There are, however, indications that the interpretation

\footnotetext{
${ }^{10}$ We note that, intriguingly, the relation between nodes resulting from the action of $X_{\phi}$ as given for the $D_{6}$-diagram in (6.2) and for the $E_{8}$-diagram in (6.5) has also been observed in 47. in an apparently unrelated context.
} 
as reflection and transmission is valid rather generically. First of all, our definition leads to the values $\mathcal{T}=0$ and $\mathcal{T}=1$ for factorising and topological defects, respectively, whose physical interpretation as fully reflective and fully transmissive is obvious. Moreover, they satisfy $\mathcal{T}+\mathcal{R}=1$ and for unitary theories take values in the interval [0,1], at least for all the examples we considered. We also showed that, in contrast to the $g$-factors [28], the quantities $\mathcal{R}$ and $\mathcal{T}$ are constant under the action of topological defects. On the other hand, as seen for the free boson and the Ising model, while $g$ is constant under (truly) marginal deformations of the defect, $\mathcal{R}$ and $\mathcal{T}$ are typically not.

Given these appealing features it is then natural to ask whether the specified bounds on $\mathcal{R}$ and $\mathcal{T}$ in unitary models can be shown to hold rigorously or whether they can finally even be improved. To this end note that if, in the notation of (2.5), the two ratios $\left\langle 0\left|\bar{L}_{2}^{i} L_{2}^{i}\right| b\right\rangle /\langle 0 \mid b\rangle$ are non-negative, i.e. if $R_{11} \geq 0$ and $R_{22} \geq 0$, then by (2.6) we have $-\omega_{b} \leq \min \left(\frac{c_{1}}{c_{2}}, \frac{c_{2}}{c_{1}}\right)$. This results in the bounds

$$
\mathcal{T} \leq \frac{2 \min \left(c_{1}, c_{2}\right)}{c_{1}+c_{2}} \quad \text { and } \quad \mathcal{R} \geq \frac{\left|c_{1}-c_{2}\right|}{c_{1}+c_{2}}
$$

These bounds hold in all unitary models we considered. In the class of examples treated in section 5.1 they can even be saturated. This happens when the chiral algebra of $\mathrm{CFT}_{1}$ (say) is contained in that of $\mathrm{CFT}_{2}$, and the defect is taken to be transmissive for all fields in this chiral algebra, so that $c=c_{1}$ in (5.5). However, we have no general proof of the assertion (7.1). Another observation worth mentioning is that the bound $\mathcal{R} \geq 0$ holds even for the non-unitary minimal models considered in section 6 .

Independent of such structural considerations the quantities $\mathcal{R}$ and $\mathcal{T}$ may be useful in the analysis of RG flows in defect systems. There are three ways to deform two conformal field theories separated by a defect: one can turn on a perturbation in the bulk of either CFT, and one can perturb the defect itself. In the last case the behaviour of the bulk theories will be unaffected. Yet, in all cases the defect will start to flow and it is an interesting question to ask what the new infrared fixed point is. The analysis of this problem is usually very hard and requires the use of numerical methods to arrive at concrete predictions 448, 49]. For pure defect flows there do exist fairly general conjectures [50, 51] for the end point of the flows. We expect the quantities $\mathcal{R}$ and $\mathcal{T}$ to be a useful tool to determine the actual fixed point in numerical calculations.

One of the initial motivations for our study of transmission amplitudes was to improve our understanding of so-called generalised permutation branes. These are conjectural nonfactorising boundary conditions in product CFTs whose constituents are structurally very close, such as minimal models with distinct central charge, or WZW (or coset) models based on the same group but at different levels. The common feature of these models is that they come in a multi-parameter family and that on a diagonal subset where two or more parameters agree one has a permutation symmetry. The latter may then be used to construct topological defects. It appears reasonable to assume that, when slightly moving away from the diagonal, these defects will not suddenly completely change their nature, in particular in the semi-classical regime where the parameters (e.g. the levels) are large and geometric reasoning applies. This picture has been confirmed in 21] in the case of product 
groups. Generalised permutation branes are also known to exist in $N=2$ topological conformal field theories [26, 27]. The only rigorous CFT construction has been performed in 22] and it is restricted to very few and rather special examples.

Despite all these activities we still miss a satisfactory answer at the moment to the question of what qualifies a defect for being associated to a 'generalised permutation brane'. Lacking any additional algebraic input, e.g. from symmetry, one could be tempted to call generalised permutation branes those branes in the families just described whose transmission can be brought arbitrarily close to one, for instance for large values of the parameters close to the diagonal. One possibility is that away from the diagonal among all possible defects those related to generalised permutation branes realise the maximal transmission coefficient that can occur for this specific choice of parameters.

In fact our investigation of non-unitary minimal models in this paper was precisely motivated by this reasoning. Indeed, in these models all the defects are known, and hence also those which are possibly connected to generalised permutation branes. In this context it is worth recalling that the Ising model possesses a relevant perturbation by an imaginary magnetic field which lets it flow to the Lee-Yang edge singularity. If we now consider the Ising model with a topological defect and switch on an imaginary magnetic field in the upper half-plane we end up with a possibly non-factorising defect which separates a Lee-Yang phase from an Ising phase and which might possess an interpretation as a generalised permutation brane. Perturbing first the upper half-plane and then the lower half plane gives a sequence of flows from Ising $\times$ Ising over Lee-Yang $\times$ Ising to Lee-Yang $\times$ LeeYang, and these are precisely the models where we determined the transmission for a complete set of conformal defects. Since the transmission and reflection do not seem to satisfy reasonable bounds in these non-unitary models and since the combined bulk/defect flows are beyond our control so far, we could not draw any conclusions regarding the interpretation of certain boundary states as generalised permutation branes. Nevertheless it appears to be a promising project to reanalyse the situation in a similar setup with unitary models, possibly in the semi-classical regime where perturbation theory is at hand.

\section{Acknowledgments}

It is a great pleasure to thank Zoltan Bajnok, Pasquale Calabrese, Jean-Sebastien Caux, Patrick Dorey, Stefan Fredenhagen and Matthias Gaberdiel for useful discussions and Jürgen Fuchs, Andreas Recknagel and Christoph Schweigert for valuable comments on the manuscript. Also, we are most grateful to the referee for pointing out references [6, 7] to us. TQ acknowledges the hospitality of the KITP in Santa Barbara during the completion of this work. This work was supported in part by the EU Research Training Network

grants 'Euclid', contract number HPRN-CT-2002-00325, 'Superstring Theory', contract number MRTN-CT-2004-512194, by the PPARC rolling grant PP/C507145/1, and by the National Science Foundation under Grant no. PHY99-07949. Until September 2006 TQ was funded by a PPARC postdoctoral fellowship under reference PPA/P/S/2002/00370. IR receives partial support from the EPSRC First Grant EP/E005047/1. 


\section{A. Reflection and Transmission via $\omega_{b}$}

In the following we give some details regarding the derivation of (2.6). Define the (anti-) holomorphic fields $W$ and $\bar{W}$ as

$$
W=c_{2} T_{1}-c_{1} T_{2} \quad, \quad \bar{W}=c_{2} \bar{T}_{1}-c_{1} \bar{T}_{2},
$$

for $c_{1}, c_{2}$ the central charges of $\mathrm{CFT}_{1}$ and $\mathrm{CFT}_{2}$. One can check that $W$ and $\bar{W}$ are primary with respect to $T^{\text {tot }}$ and $\bar{T}^{\text {tot }}$, and that the OPE of $W$ with itself reads

$$
W(z) W(w)=\frac{\frac{1}{2} c_{1} c_{2}\left(c_{1}+c_{2}\right)}{(z-w)^{4}}+\left(\frac{2}{(z-w)^{2}}+\frac{1}{z-w} \frac{\partial}{\partial w}\right)\left(\left(c_{2}-c_{1}\right) W(w)+c_{1} c_{2} T^{\mathrm{tot}}(w)\right)+\cdots
$$

For a boundary state $|b\rangle$ we have

$$
\left\langle 0\left|W_{2} \bar{L}_{2}^{\text {tot }}\right| b\right\rangle=0 \quad \text { and } \quad\left\langle 0\left|L_{2}^{\text {tot }} \bar{W}_{2}\right| b\right\rangle=0
$$

since e.g. $\left\langle 0\left|W_{2} \bar{L}_{2}^{\text {tot }}\right| b\right\rangle=\left\langle 0\left|W_{2} L_{-2}^{\text {tot }}\right| b\right\rangle$ and $\langle 0| W_{2} L_{-m}^{\text {tot }}=0$ for $m>0$ as $W$ is primary. To proceed we need to assume that

$$
c_{1} \neq 0, \quad c_{2} \neq 0 \quad \text { and } \quad c_{1}+c_{2} \neq 0
$$

Using the conditions (A.3), written out in terms of the $L_{m}^{1,2}$ and $\bar{L}_{m}^{1,2}$, it is straightforward to verify the identities

$$
\begin{aligned}
& \left\langle 0\left|L_{2}^{1} \bar{L}_{2}^{1}\right| b\right\rangle=\frac{1}{\left(c_{1}+c_{2}\right)^{2}}\left(c_{1}^{2}\left\langle 0\left|L_{2}^{\mathrm{tot}} \bar{L}_{2}^{\mathrm{tot}}\right| b\right\rangle+\left\langle 0\left|W_{2} \bar{W}_{2}\right| b\right\rangle\right) \\
& \left\langle 0\left|L_{2}^{2} \bar{L}_{2}^{2}\right| b\right\rangle=\frac{1}{\left(c_{1}+c_{2}\right)^{2}}\left(c_{2}^{2}\left\langle 0\left|L_{2}^{\mathrm{tot}} \bar{L}_{2}^{\mathrm{tot}}\right| b\right\rangle+\left\langle 0\left|W_{2} \bar{W}_{2}\right| b\right\rangle\right) \\
& \left\langle 0\left|L_{2}^{1} \bar{L}_{2}^{2}\right| b\right\rangle=\left\langle 0\left|L_{2}^{2} \bar{L}_{2}^{1}\right| b\right\rangle=\frac{1}{\left(c_{1}+c_{2}\right)^{2}}\left(c_{1} c_{2}\left\langle 0\left|L_{2}^{\mathrm{tot}} \bar{L}_{2}^{\mathrm{tot}}\right| b\right\rangle-\left\langle 0\left|W_{2} \bar{W}_{2}\right| b\right\rangle\right)
\end{aligned}
$$

Of course we can also write $\left\langle 0\left|L_{2}^{\text {tot }} \bar{L}_{2}^{\text {tot }}\right| b\right\rangle=\left\langle 0\left|L_{2}^{\text {tot }} L_{-2}^{\text {tot }}\right| b\right\rangle=\frac{1}{2}\left(c_{1}+c_{2}\right)\langle 0 \mid b\rangle$. To obtain (2.6) we just need to introduce

$$
\omega_{b}=\frac{2}{c_{1} c_{2}\left(c_{1}+c_{2}\right)} \cdot \frac{\left\langle 0\left|W_{2} \bar{W}_{2}\right| b\right\rangle}{\langle 0 \mid b\rangle}
$$

use this to replace $\left\langle 0\left|W_{2} \bar{W}_{2}\right| b\right\rangle$ in $($ A.5 $)$ and divide both sides by $\langle 0 \mid b\rangle$.

Note that for a factorising boundary condition $|b\rangle=\left|b_{1}\right\rangle \otimes\left|b_{2}\right\rangle$, the inner product $\left\langle 0\left|L_{2}^{1} \bar{L}_{2}^{2}\right| b\right\rangle$ will be zero, i.e. the entry $R_{12}$ of the matrix $R$ in (2.6) is zero. Together with our assumptions (A.4) on the central charges this implies

$$
\omega_{b}=1 \text { for factorising defects }
$$

On the other hand, for a permutation boundary condition (corresponding to a topological defect in the unfolded model), we have $\left\langle 0\left|L_{2}^{1} \bar{L}_{2}^{1}\right| b\right\rangle=\left\langle 0\left|L_{2}^{1} L_{-2}^{2}\right| b\right\rangle=0$. Since furthermore 
the existence of topological defects in the unfolded model implies $c_{1}=c_{2}$, together with (2.6) we obtain

$$
\omega_{b}=-1 \text { for topological defects . }
$$

Finally, instead of the exterior of the unit circle, consider the folded CFT on the upper half-plane with boundary condition $b$. Since the boundary condition is conformal, we have

$T^{\text {tot }}(x)=\bar{T}^{\text {tot }}(x)$ for points $x$ on the real axis. From the definition (A.1) it is then not difficult to see that

$$
W(x)=\bar{W}(x) \text { for all real } x \text { if and only if } b \text { is factorising . }
$$

\section{B. Summary on states in the coset decomposition}

In this appendix we present the bulky formulas for the states that are relevant in section 5.2 of this note. We will use the spin basis in which the generators of the affine $S U(2)_{k}$ satisfy

$$
\left[E_{m}^{+}, E_{n}^{-}\right]=k \delta_{m+n, 0}+2 H_{m+n},\left[H_{m}, E_{n}^{ \pm}\right]= \pm E_{m+n}^{ \pm},\left[H_{m}, H_{n}\right]=\frac{k}{2} \delta_{m+n, 0} .
$$

The affine highest weight states $|j\rangle$ are introduced as usual by demanding

$$
H_{0}|j\rangle=j|j\rangle, \quad E_{0}^{+}|j\rangle=0, \quad E_{n}^{ \pm}|j\rangle=H_{n}|j\rangle=0 \text { for } n>0 .
$$

With these definitions it is then a straightforward exercise to come up with the following set of orthonormalised singlet states

$$
\begin{aligned}
& \left|v_{1}\right\rangle=\sqrt{\frac{2}{c_{k_{1}+k_{2}}}}|(0,0,0)\rangle \otimes L_{-2}|0\rangle \\
& \left|v_{2}\right\rangle=\sqrt{\frac{2}{c_{k_{1}}+c_{k_{2}}-c_{k_{1}+k_{2}}}} L_{-2}|(0,0,0)\rangle \otimes|0\rangle \\
& \left|v_{3}\right\rangle=\frac{1}{4 \sqrt{3\left(k_{1}+k_{2}+4\right)}}|(0,0,1)\rangle \otimes\left(4 E_{-1}^{-}-H_{-1} E_{0}^{-}-2 E_{-1}^{+} E_{0}^{-} E_{0}^{-}\right)|1\rangle
\end{aligned}
$$

in the decomposed theory (5.7) which are relevant for the description of the states $L_{-2}^{i}|0\rangle$ appearing in eq. (2.5).

Using the concrete form of the Sugawara and GKO energy momentum tensors and the definition of the state $|(0,0,1)\rangle \otimes|1\rangle$ (see (5.11) ) one can easily show that the linear combinations relevant to a solution of our problem are given by

$$
\begin{aligned}
L_{-2}^{1}|0\rangle= & \sqrt{\frac{3 k_{1}^{2}}{2\left(k_{1}+k_{2}\right)\left(k_{1}+k_{2}+2\right)}}\left|v_{1}\right\rangle+\sqrt{\frac{3 k_{1} k_{2}\left(k_{2}+2\right)}{2\left(k_{1}+2\right)\left(k_{1}+k_{2}+2\right)\left(k_{1}+k_{2}+4\right)}}\left|v_{2}\right\rangle \\
& +\sqrt{\frac{3 k_{1} k_{2}}{\left(k_{1}+k_{2}\right)\left(k_{1}+k_{2}+4\right)}}\left|v_{3}\right\rangle
\end{aligned}
$$




$$
\begin{aligned}
L_{-2}^{2}|0\rangle= & \sqrt{\frac{3 k_{2}^{2}}{2\left(k_{1}+k_{2}\right)\left(k_{1}+k_{2}+2\right)}}\left|v_{1}\right\rangle+\sqrt{\frac{3 k_{1} k_{2}\left(k_{1}+2\right)}{2\left(k_{2}+2\right)\left(k_{1}+k_{2}+2\right)\left(k_{1}+k_{2}+4\right)}}\left|v_{2}\right\rangle \\
& -\sqrt{\frac{3 k_{1} k_{2}}{\left(k_{1}+k_{2}\right)\left(k_{1}+k_{2}+4\right)}}\left|v_{3}\right\rangle \\
L_{-2}^{\text {tot }}|0\rangle= & |(0,0,0)\rangle \otimes L_{-2}|0\rangle+L_{-2}|(0,0,0)\rangle \otimes|0\rangle \\
= & \sqrt{\frac{c_{k_{1}+k_{2}}}{2}}\left|v_{1}\right\rangle+\sqrt{\frac{c_{k_{1}}+c_{k_{2}}-c_{k_{1}+k_{2}}}{2}}\left|v_{2}\right\rangle .
\end{aligned}
$$

Identical relations hold for the anti-holomorphic sector. Combining both sectors we thus find

$$
\begin{aligned}
L_{-2}^{1}|0\rangle \otimes \bar{L}_{-2}^{1} \overline{|0\rangle}= & \frac{3 k_{1}^{2}}{2\left(k_{1}+k_{2}\right)\left(k_{1}+k_{2}+2\right)}\left|v_{1}\right\rangle \otimes \overline{\left|v_{1}\right\rangle} \\
& +\frac{3 k_{1} k_{2}\left(k_{2}+2\right)}{2\left(k_{1}+2\right)\left(k_{1}+k_{2}+2\right)\left(k_{1}+k_{2}+4\right)}\left|v_{2}\right\rangle \otimes \overline{\left|v_{2}\right\rangle} \\
& +\frac{3 k_{1} k_{2}}{\left(k_{1}+k_{2}\right)\left(k_{1}+k_{2}+4\right)}\left|v_{3}\right\rangle \otimes \overline{\left|v_{3}\right\rangle}+\text { mixed contributions } \\
L_{-2}^{2}|0\rangle \otimes \bar{L}_{-2}^{2} \overline{|0\rangle}= & \frac{3 k_{2}^{2}}{2\left(k_{1}+k_{2}\right)\left(k_{1}+k_{2}+2\right)}\left|v_{1}\right\rangle \otimes \overline{\left|v_{1}\right\rangle} \\
& +\frac{3 k_{1} k_{2}\left(k_{1}+2\right)}{2\left(k_{2}+2\right)\left(k_{1}+k_{2}+2\right)\left(k_{1}+k_{2}+4\right)}\left|v_{2}\right\rangle \otimes \overline{\left|v_{2}\right\rangle} \\
& +\frac{3 k_{1} k_{2}}{\left(k_{1}+k_{2}\right)\left(k_{1}+k_{2}+4\right)}\left|v_{3}\right\rangle \otimes \overline{\left|v_{3}\right\rangle}+\text { m.c. } \\
L_{-2}^{1}|0\rangle \otimes \bar{L}_{-2}^{2} \overline{|0\rangle=} & \frac{3 k_{1} k_{2}}{2\left(k_{1}+k_{2}\right)\left(k_{1}+k_{2}+2\right)}\left|v_{1}\right\rangle \otimes \overline{\left|v_{1}\right\rangle} \\
& +\frac{3 k_{1} k_{2}}{2\left(k_{1}+k_{2}+2\right)\left(k_{1}+k_{2}+4\right)}\left|v_{2}\right\rangle \otimes \overline{\left|v_{2}\right\rangle} \\
& -\frac{3 k_{1} k_{2}}{\left(k_{1}+k_{2}\right)\left(k_{1}+k_{2}+4\right)}\left|v_{3}\right\rangle \otimes \overline{\left|v_{3}\right\rangle}+\text { m.c. } \\
L_{-2}^{\text {tot }}|0\rangle \otimes \bar{L}_{-2}^{\text {tot }} \overline{|0\rangle}= & \frac{c_{k_{1}+k_{2}}\left|v_{1}\right\rangle \otimes \overline{\left|v_{1}\right\rangle}+\frac{c_{k_{1}}+c_{k_{2}}-c_{k_{1}+k_{2}}}{2}\left|v_{2}\right\rangle \otimes \overline{\left|v_{2}\right\rangle}+\text { m.c. } .}{2}
\end{aligned}
$$

The mixed contributions "m.c." have vanishing overlap with Ishibashi states and have therefore not been spelled out.

\section{Category theoretic calculations}

In this appendix we will briefly describe the calculations behind the results stated in section 6. We will make use of the 'topological field theory approach to rational conformal field theory', or TFT-approach for short, developed in [52, 14, 53, 54]. Rather than reviewing 
this formalism, we highlight a few points in the next section, and also provide references to the relevant parts of these papers where more details can be found.

\section{C.1 Preliminaries}

In the TFT approach one starts from a rational chiral algebra $\mathcal{V}$ (a conformal vertex algebra) and considers its representation category $\mathcal{C}=\operatorname{Rep}(\mathcal{V})$. For Virasoro minimal models, $\mathcal{V}$ is the Virasoro vertex algebra at the corresponding central charge, and the simple objects in $\mathcal{C}$ are labelled by entries of the Kac table (modulo its $\mathbb{Z}_{2}$-symmetry).

One of the properties one demands of a rational CFT is that the category $\mathcal{C}$ should be modular, i.e. (roughly) a $\mathbb{C}$-linear semi-simple braided tensor category, where the braiding obeys a certain non-degeneracy condition (see e.g. [54, app. A.1] for details and references). Given a modular tensor category $\mathcal{C}$, one can construct a three-dimensional topological field theory (TFT), which has the chiral CFT described by $\mathcal{V}$ as its boundary degrees of freedom (see [54, app. A.2] and references therein).

A full conformal field theory that includes $\mathcal{V}$ in its chiral symmetries can now be described by a symmetric special Frobenius algebra $A$ in the category $\mathcal{C}$ (see [54, app. B] for a summary). Let us denote this conformal field theory by $\mathrm{CFT}_{A}$. Properties of $\mathrm{CFT}_{A}$, like boundary conditions (preserving $\mathcal{V}$ with trivial gluing automorphism) or topological defects transparent to the fields in $\mathcal{V}$, are related to natural quantities obtained from $A$, like modules and bimodules, respectively (see [14, sect. 4.4]). Structure constants of the CFT are expressed as invariants of framed links in a three-manifold; the value of these invariants is computed via the TFT defined by $\mathcal{C}$ [53].

\section{C.1.1 Induced modules}

As already mentioned, modules of $A$ describe boundary conditions of $\mathrm{CFT}_{A}$, and simple modules correspond to elementary boundary conditions. The simplest class of $A$-modules are the induced modules. Given an object $U$ of $\mathcal{C}$, the induced module $\operatorname{Ind}_{A}(U)$ is simply $A \otimes U$ with the action of $A$ given by the multiplication of $A$. One can show that in the present setting, every simple module is a submodule of an induced module (see 14, sect. 4.3] for details and references). So to find all simple $A$-modules we have to decompose all induced modules based on simple objects of $\mathcal{C}$. For two $A$-modules $M, N$ denote by $\operatorname{Hom}_{A}(M, N)$ the subspace of morphisms in $\mathcal{C}$ from $M$ to $N$, which commute with the action of $A$. The following property is very useful when decomposing induced modules,

$$
\operatorname{Hom}_{A}\left(\operatorname{Ind}_{A}(U), M\right) \cong \operatorname{Hom}(U, M)
$$

where the second Hom-space denotes morphisms in $\mathcal{C}$. In particular, if $\operatorname{Hom}_{A}\left(\operatorname{Ind}_{A}(U)\right.$, $\left.\operatorname{Ind}_{A}(U)\right) \cong \operatorname{Hom}(U, A \otimes U)$ is one-dimensional, then $\operatorname{Ind}_{A}(U)$ is simple, and if $\operatorname{Hom}(A \otimes U, U)$ is two-dimensional, then $\operatorname{Ind}_{A}(U)$ decomposes into two non-isomorphic simple $A$-modules.

We will denote the boundary condition of $\mathrm{CFT}_{A}$ labelled by an $A$-module $M$ by $B_{M}$.

\section{C.1.2 Local modules and factorising defects}

In (A.9) we have seen that a boundary condition of the folded model (or a defect line in the unfolded model) is factorising if and only if $W=\bar{W}$ on the real axis. In the minimal 
model examples treated below, the algebra $A$ describes the extension of the symmetry from the Virasoro algebra at central charge $c_{1}+c_{2}$ to the product of the two Virasoro (vertex) algebras with central charges $c_{1}$ and $c_{2}$. In particular, this extension contains the primary field $W$. In terms of the representation category $\mathcal{C}$ this means that the representation $U_{W}$ of the chiral algebra which contains the field $W$, is a subobject of $A$. Also, if $A$ describes an extended symmetry, it will be a commutative special symmetric Frobenius algebra. So from here on we will assume that $A$ is commutative. Note that from the definition given above, $A$, seen as a left module over itself, is local.

Recall that $\mathcal{C}$ is in particular a braided tensor category; the braiding is denoted by $c_{U V} \in \operatorname{Hom}(U \otimes V, V \otimes U)$. Let $M$ be an $A$-module and let $\rho_{M} \in \operatorname{Hom}(A \otimes M, M)$ be the representation morphism (see [54, app. A.4] for details). The module $M$ is called local iff $\rho_{M} \circ c_{M A} \circ c_{A M}=\rho_{M}$. One can show that a simple module $M$ is local if and only if all irreducible representations of the chiral algebra entering $M$ have the same conformal weight modulo $\mathbb{Z}$ (i.e. if the twist on $M$ is a multiple of the identity, see e.g. [55, corollary 3.18] for a proof).

As explained in [53, sect 3.3], the multiplicity space of bulk fields of $\mathrm{CFT}_{A}$ transforming in the representation $U_{i} \times U_{j}$ of the left/right copy of the chiral symmetry is isomorphic to a certain morphism space $\operatorname{Hom}_{A \mid A}\left(U_{i} \otimes^{+} A \otimes^{-} U_{j}, A\right)$. If we pick an embedding $e_{W} \in$ $\operatorname{Hom}\left(U_{W}, A\right)$, we can take the chiral field $W$ to correspond to the morphism $\phi_{W}=m \circ$ $\left(e_{W} \otimes \operatorname{id}_{A}\right) \in \operatorname{Hom}_{A \mid A}\left(U_{W} \otimes^{+} A \otimes^{-} \mathbf{1}, A\right)$ (this uses that $A$ is commutative), and similarly the anti-chiral field $\bar{W}(z)$ corresponds to $\phi_{\bar{W}}=m \circ\left(\mathrm{id}_{A} \otimes e_{W}\right) \in \operatorname{Hom}_{A \mid A}\left(\mathbf{1} \otimes^{+} A \otimes^{-} U_{W}, A\right)$.

From the ribbon graph representation [53, eqn. (4.15)] of an upper half-plane correlator involving $W$ or $\bar{W}$, and a boundary condition $B_{M}$ labelled by the module $M$, it is not too difficult to deduce that

$$
M \text { local } \quad \Rightarrow \quad B_{M} \text { is factorising boundary condition . }
$$

The converse holds if $A$ is generated by the subobject $U_{W}$ in an appropriate sense, but we

will not need this here. Note also that since $A$ is a local module over itself (we assumed $A$ to be commutative), it describes a factorising boundary condition.

\section{C.1.3 Computing the coefficient $\omega$}

Denote by $|M\rangle$ the boundary state described by an $A$-module $M$, and by $|U\rangle\rangle$ the Ishibashi state belonging to the simple object $U$ (which is an irreducible representation of the chiral algebra). As usual one can write the boundary state as a sum of Ishibashi states, and accordingly there are constants $C\left(U_{W} ; M\right)$ such that

$$
\left.\left\langle 0\left|W_{2} \bar{W}_{2}\right| M\right\rangle=C\left(U_{W} ; M\right)\langle 0 \mid A\rangle \cdot\left\langle 0\left|W_{2} \bar{W}_{2}\right| U_{W}\right\rangle\right\rangle
$$

where we chose to normalise the coefficients $C\left(U_{W} ; M\right)$ relative to $\langle 0 \mid A\rangle$, the inner product of the out-vacuum with the boundary state corresponding to $A$. In the TFT approach, $C\left(U_{W} ; M\right)$ can be expressed as the invariant of a ribbon graph. If, similar to the previous section, we take the morphism in $\operatorname{Hom}_{A \mid A}\left(U_{W} \otimes^{+} A \otimes^{-} U_{W}, A\right)$ corresponding to the nonchiral field $W \bar{W}$ to be $\phi_{W \bar{W}}=m \circ\left(m \otimes \mathrm{id}_{A}\right) \circ\left(e_{W} \otimes \mathrm{id}_{A} \otimes e_{W}\right)$, then according to [53, 
eqn. (4.20)] the relevant invariant is

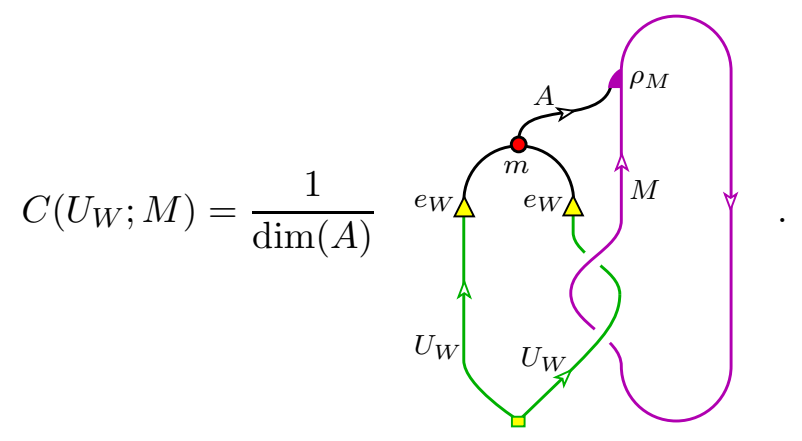

By $\operatorname{dim}(\cdot)$ we denote the quantum dimension of an object in $\mathcal{C}$. The constants (C.4 have the property $C(\mathbf{1} ; A)=1$, as they should have for C.3 to hold (we take the Ishibashi state $|\mathbf{1}\rangle\rangle$ to be normalised as $\langle 0 \mid \mathbf{1}\rangle\rangle=1$ ). Inserting (C.3) into the definition (A.6) of $\omega$ gives, for the boundary condition $B_{M}$,

$$
\omega_{M}=\frac{\left.2\left\langle 0\left|W_{2} \bar{W}_{2}\right| U_{W}\right\rangle\right\rangle}{c_{1} c_{2}\left(c_{1}+c_{2}\right)} \cdot \frac{\langle 0 \mid A\rangle}{\langle 0 \mid M\rangle} \cdot C\left(U_{W} ; M\right) .
$$

Since we know that $A$ corresponds to a factorising boundary condition, we conclude

$$
1=\omega_{A}=\frac{\left.2\left\langle 0\left|W_{2} \bar{W}_{2}\right| U_{W}\right\rangle\right\rangle}{c_{1} c_{2}\left(c_{1}+c_{2}\right)} \cdot C\left(U_{W} ; A\right)
$$

This, together with $\langle 0 \mid A\rangle /\langle 0 \mid M\rangle=\operatorname{dim}(A) / \operatorname{dim}(M)$ (which follows as a special case from [53, sect.4.3]) allows one to simplify (C.5) to

$$
\omega_{M}=\frac{\operatorname{dim}(A)}{\operatorname{dim}(M)} \cdot \frac{C\left(U_{W} ; M\right)}{C\left(U_{W} ; A\right)}
$$

This expression is now defined entirely in terms of data accessible on the level of the category $\mathcal{C}$ (as opposed to (C.5) which e.g. makes explicit mention of the central charges). If furthermore $M$ is an induced module $\operatorname{Ind}_{A}(V)$ for some object $V$, then it is not too difficult to see that (C.7) simplifies even further, namely to

$$
\omega_{\operatorname{Ind}_{A}(V)}=\frac{s_{U_{W}, V}}{\operatorname{dim}(W) \operatorname{dim}(V)},
$$

where $s_{U, V}$ denotes the invariant of the Hopf-link. It is related to the modular $S$-matrix occurring in the transformation of characters via $s_{U_{i}, U_{j}}=S_{i j} / S_{00}$ for two simple objects $U_{i}, U_{j}$. The quantum dimension in turn can be expressed as $\operatorname{dim}\left(U_{i}\right)=S_{i 0} / S_{00}$.

\section{C.1.4 Action of topological defects}

The properties of topological defects, and how they can be calculated in the TFT approach, has been discussed in detail in [17]. Bimodules of $A$ describe topological defects, and simple bimodules correspond to elementary topological defects. As described in section 2 , topological defects can be fused. In terms of the $A$-bimodules, the corresponding operation is the tensor product over $A$. That is, if $Y, Z$ are $A$-bimodules, and $X_{Y}, X_{Z}$ are the 
corresponding defects, then $X_{Y} \star X_{Z}=X_{Y \otimes_{A} Z}$. The same holds for the action on boundary conditions. Given an $A$-module $M$, one obtains $X_{Y} \star B_{M}=B_{Y \otimes_{A} M}$.

For models with charge conjugation modular invariant, like the Lee-Yang, Ising, and $M_{2,7}$ minimal models treated below, conformal boundary conditions and topological defects are labelled by the irreducible representations and their fusion is just given by the fusion product of the corresponding representations [9, 10]. In the present context that follows since the relevant algebra in this case is simply $A=\mathbf{1}$.

On the other hand, to understand the defect action for the $D_{6}, E_{6}$ and $E_{8}$-type modular invariants appearing below, we need to evaluate the tensor products over the relevant algebra $A$. To this end we need two additional concepts: $\alpha$-induced bimodules and a procedure to turn a left module into a bimodule. Given an object $U$ of $\mathcal{C}$, there are two natural ways to define a bimodule structure on the object $A \otimes U$. Just as for induced left modules, for the left action we simply use the multiplication on $A$. For the right action, we need to take the object $A$ past $U$ before we can use the multiplication. Since $\mathcal{C}$ is braided, there are two ways to do so (via over and under braiding), and we denote the resulting bimodules by $\alpha_{A}^{+}(U)$ and $\alpha_{A}^{-}(U)$ (see e.g. [55, def. 2.21] for more details and references). If the two bimodules happen to be isomorphic (as will be the case for the $\alpha$ induced bimodules considered below), we just write $\alpha_{A}(U)$. In the present application, the algebra $A$ is commutative. In this case, every left module $M$ carries two natural bimodules structures. The right action is defined by first using the over or under braiding to take $A$ past $M$, and then applying the left representation morphism $\rho_{M}$ (in verifying that this is a right action one needs $A$ to be commutative). If the module $M$ is local, it is easy to see that these two bimodule structures coincide, and we will denote the resulting bimodule as $M^{\text {bi }}$. One can check that, if $A$ is commutative and the induced module $\operatorname{Ind}_{A}(U)$ is local, then $\operatorname{Ind}_{A}(U)^{\mathrm{bi}}=\alpha_{A}(U)$. To compute the fusion of defects and boundary conditions, the following rule is useful,

$$
\alpha_{A}^{ \pm}(U) \otimes_{A} \operatorname{Ind}_{A}(V) \cong \operatorname{Ind}_{A}(U \otimes V)
$$

where $U$ and $V$ are objects of $\mathcal{C}$. Writing out the definitions of $\alpha_{A}^{ \pm}(U)$ and $\operatorname{Ind}_{A}(V)$, it is straightforward to give an explicit isomorphism in terms of the braiding of $\mathcal{C}$.

\section{C.2 Lee-Yang $\times$ Lee-Yang}

The product of two $M_{2,5}$ minimal models can be described as the $D_{6}$ invariant of $M_{3,10}$. The Kac tables of these minimal models are:

\begin{tabular}{c||c|c|c|c|}
\multicolumn{5}{c}{$M_{2,5}$} \\
\hline 1 & 0 & $-\frac{1}{5}$ & $-\frac{1}{5}$ & 0 \\
\hline \hline & 1 & 2 & 3 & 4
\end{tabular}

\begin{tabular}{c||c|c|c|c|c|c|c|c|c|}
\multicolumn{10}{c|}{$M_{3,10}$} \\
\hline 2 & 2 & $\frac{49}{40}$ & $\frac{3}{5}$ & $\frac{1}{8}$ & $-\frac{1}{5}$ & $-\frac{3}{8}$ & $-\frac{2}{5}$ & $-\frac{11}{40}$ & 0 \\
\hline 1 & 0 & $-\frac{11}{40}$ & $-\frac{2}{5}$ & $-\frac{3}{8}$ & $-\frac{1}{5}$ & $\frac{1}{8}$ & $\frac{3}{5}$ & $\frac{49}{40}$ & 2 \\
\hline \hline & 1 & 2 & 3 & 4 & 5 & 6 & 7 & 8 & 9
\end{tabular}


Let us denote the irreducible representation of $M_{3,10}$ with Kac label $(r, s)$ by $U_{r, s}$. Then the algebra $A$ is given by $A=U_{1,1} \oplus U_{1,9}$ (as an object, we do not need here the explicit form of the multiplication), and $W$ is the primary state in the representation $U_{W}=U_{1,9}$.

By the method of induced modules and (C.1) one finds that $\operatorname{Ind}_{A}\left(U_{1,1}\right), \operatorname{Ind}_{A}\left(U_{1,2}\right)$, $\operatorname{Ind}_{A}\left(U_{1,3}\right), \operatorname{Ind}_{A}\left(U_{1,4}\right)$ are simple and that $\operatorname{Ind}_{A}\left(U_{1,5}\right)=M_{+} \oplus M_{-}$for two simple $A$ modules $M_{ \pm}$. These are all simple modules of $A$. As objects they decompose as

$$
\begin{aligned}
& \operatorname{Ind}_{A}\left(U_{1,1}\right)=U_{1,1} \oplus U_{1,9} \quad, \quad \operatorname{Ind}_{A}\left(U_{1,2}\right)=U_{1,2} \oplus U_{1,8} \quad, \\
& \operatorname{Ind}_{A}\left(U_{1,3}\right)=U_{1,3} \oplus U_{1,7} \quad, \quad \operatorname{Ind}_{A}\left(U_{1,4}\right)=U_{1,4} \oplus U_{1,6} \quad, \quad M_{ \pm}=U_{1,5} .
\end{aligned}
$$

A glance at the conformal weights $\bmod \mathbb{Z}$ reveals that $\operatorname{Ind}_{A}\left(U_{1,1}\right), \operatorname{Ind}_{A}\left(U_{1,3}\right)$ and $M_{ \pm}$are local, and, denoting the representations of $M_{2,5}$ by $\mathbf{1}$ (for weight 0 ) and $\phi$ (for weight $-\frac{1}{5}$ ), that in terms of the product model

$$
\operatorname{Ind}_{A}\left(U_{1,1}\right)=\mathbf{1} \times \mathbf{1}, \quad \operatorname{Ind}_{A}\left(U_{1,3}\right)=\phi \times \phi, \quad M_{+}=\phi \times \mathbf{1}, \quad M_{-}=\mathbf{1} \times \phi,
$$

where the last two identifications are a choice of convention.

To see the $D_{6}$ diagram appear we have to draw a dot for each boundary condition and draw a line between two dots whenever the representation $U_{1,2}$ appears in the space of states for the strip with these two boundary conditions on either side [45, 46]. In the present language this means we draw a dot for each $A$-module, and given two $A$-modules $M, N$ we draw $\operatorname{dim} \operatorname{Hom}_{A}\left(M \otimes U_{1,2}, N\right)$ lines between them. Using that for induced modules $\operatorname{Ind}_{A}(U) \otimes V=\operatorname{Ind}_{A}(U \otimes V)$ one finds

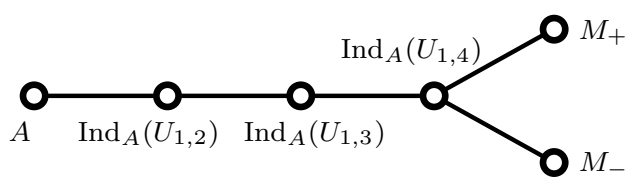

For example, $\operatorname{dim} \operatorname{Hom}_{A}\left(\operatorname{Ind}_{A}\left(U_{1,4}\right) \otimes U_{1,2}, M_{+}\right)=\operatorname{dim} \operatorname{Hom}\left(U_{1,4} \otimes U_{1,2}, U_{1,5}\right)=1$.

We know that the product model has four factorising boundary conditions and two permutation boundary conditions. This already accounts for all six conformal boundary conditions of the $D$-invariant of $M_{3,10}$. The local modules give factorising boundary conditions, so that the two permutation ones correspond to the boundary conditions $B_{\operatorname{Ind}_{A}\left(U_{1,2}\right)}$ and $B_{\operatorname{Ind}_{A}\left(U_{1,4}\right)}$. To identify which is which, we compute the action of the topological defect $X_{\phi} \times X_{\phi}$ on the boundary condition $B_{\operatorname{Ind}_{A}\left(U_{1,2}\right)}$. Expressing this in terms of bimodules, one finds $\alpha_{A}\left(U_{1,3}\right) \otimes_{A} \operatorname{Ind}_{A}\left(U_{1,2}\right) \cong \operatorname{Ind}_{A}\left(U_{1,2}\right) \oplus \operatorname{Ind}_{A}\left(U_{1,4}\right)$. The fact that this is a direct sum of two simple modules, rather than four, is only consistent with

$$
B_{\operatorname{Ind}_{A}\left(U_{1,2}\right)}=\text { folded defect } X_{1} \quad, \quad B_{\operatorname{Ind}_{A}\left(U_{1,4}\right)}=\text { folded defect } X_{\phi} .
$$

The action of the topological defects of $M_{2,5} \times M_{2,5}$ on all boundary conditions listed in (C.12) is now simply computed by noting that for $A$-series models the fusion of a defect with another defect or a boundary condition agrees with the fusion of the chiral representations labelling them. From the identifications (C.11) and (C.13) we then conclude, for example, $\left(X_{\phi} \times X_{1}\right) \star B_{M_{-}}=B_{\operatorname{Ind}_{A}\left(U_{1,3}\right)}$ or $\left(X_{1} \times X_{\phi}\right) \star B_{\operatorname{Ind}_{A}\left(U_{1,4}\right)}=B_{\operatorname{Ind}_{A}\left(U_{1,2}\right)}+B_{\operatorname{Ind}_{A}\left(U_{1,4}\right)}$. In this way one can obtain all the data presented in (6.2). 


\section{C.3 Lee-Yang $\times$ Ising}

The product $M_{2,5} \times M_{3,4}$ is equivalent to the $E_{6}$-invariant of $M_{5,12}$. The Kac table of $M_{2,5}$ was given in the previous section, and those of $M_{3,4}$ and $M_{5,12}$ are:

\begin{tabular}{c||c|c|c|}
\multicolumn{4}{c}{$M_{3,4}$} \\
\hline 2 & $\frac{1}{2}$ & $\frac{1}{16}$ & 0 \\
\hline 1 & 0 & $\frac{1}{16}$ & $\frac{1}{2}$ \\
\hline \hline & 1 & 2 & 3
\end{tabular}

\begin{tabular}{c||c|c|c|c|c|c|c|c|c|c|c|}
\multicolumn{10}{c|}{$M_{5,12}$} \\
\hline 4 & $\frac{15}{2}$ & $\frac{93}{16}$ & $\frac{13}{3}$ & $\frac{49}{16}$ & 2 & $\frac{55}{48}$ & $\frac{1}{2}$ & $\frac{1}{16}$ & $-\frac{1}{6}$ & $-\frac{3}{16}$ & 0 \\
\hline 3 & $\frac{19}{5}$ & $\frac{209}{80}$ & $\frac{49}{30}$ & $\frac{69}{80}$ & $\frac{3}{10}$ & $\frac{-13}{240}$ & $-\frac{1}{5}$ & $-\frac{11}{80}$ & $\frac{2}{15}$ & $\frac{49}{80}$ & $\frac{13}{10}$ \\
\hline 2 & $\frac{13}{10}$ & $\frac{49}{80}$ & $\frac{2}{15}$ & $-\frac{11}{80}$ & $-\frac{1}{5}$ & $\frac{-13}{240}$ & $\frac{3}{10}$ & $\frac{69}{80}$ & $\frac{49}{30}$ & $\frac{209}{80}$ & $\frac{19}{5}$ \\
\hline 1 & 0 & $-\frac{3}{16}$ & $-\frac{1}{6}$ & $\frac{1}{16}$ & $\frac{1}{2}$ & $\frac{55}{48}$ & 2 & $\frac{49}{16}$ & $\frac{13}{3}$ & $\frac{93}{16}$ & $\frac{15}{2}$ \\
\hline \hline & 1 & 2 & 3 & 4 & 5 & 6 & 7 & 8 & 9 & 10 & 11
\end{tabular}

To have a unique labelling of the irreducible representations of $M_{5,12}$ we restrict to the range of Kac labels $(r, s)$ for which $r \in\{1,3\}$. Also, as before, by $\mathbf{1}$ and $\phi$ we will denote the two irreducible representations for $M_{2,5}$. For the Ising model $M_{3,4}$ we choose the labels 1, $\sigma$ and $\varepsilon$ for the representations of highest weight $0, \frac{1}{16}$ and $\frac{1}{2}$, respectively.

The primary field $W$ is the highest weight state of the representation $U_{W}=U_{1,7}$, and the relevant commutative Frobenius algebra is built on the object $A=U_{1,1} \oplus U_{1,7}$. Next we work out how the induced $A$-modules decompose into simple modules. For $r \in\{1,3\}$, the result is

$$
\begin{array}{lll}
\operatorname{Ind}_{A}\left(U_{r, 1}\right)(\text { simple }) & \operatorname{Ind}_{A}\left(U_{r, 7}\right) \cong \operatorname{Ind}_{A}\left(U_{r, 1}\right) \oplus \operatorname{Ind}_{A}\left(U_{r, 3}\right) \\
\operatorname{Ind}_{A}\left(U_{r, 2}\right)(\text { simple }) & \operatorname{Ind}_{A}\left(U_{r, 8}\right) \cong \operatorname{Ind}_{A}\left(U_{r, 2}\right) \oplus M_{r} \\
\operatorname{Ind}_{A}\left(U_{r, 3}\right)(\operatorname{simple}) & \operatorname{Ind}_{A}\left(U_{r, 9}\right) \cong \operatorname{Ind}_{A}\left(U_{r, 3}\right) \\
\operatorname{Ind}_{A}\left(U_{r, 4}\right) \cong \operatorname{Ind}_{A}\left(U_{r, 10}\right) \oplus M_{r} & \operatorname{Ind}_{A}\left(U_{r, 10}\right)(\operatorname{simple}) \\
\operatorname{Ind}_{A}\left(U_{r, 5}\right) \cong \operatorname{Ind}_{A}\left(U_{r, 3}\right) \oplus \operatorname{Ind}_{A}\left(U_{r, 11}\right) & \operatorname{Ind}_{A}\left(U_{r, 11}\right)(\operatorname{simple}) \\
\operatorname{Ind}_{A}\left(U_{r, 6}\right) \cong \operatorname{Ind}_{A}\left(U_{r, 2}\right) \oplus \operatorname{Ind}_{A}\left(U_{r, 10}\right) &
\end{array}
$$

For each value of $r \in\{1,3\}$ there are six simple modules: the induced modules for the objects $U_{r, 1}, U_{r, 2}, U_{r, 3}, U_{r, 10}, U_{r, 11}$, and the module $M_{r}$. The Dynkin diagram is again found by computing $\operatorname{dim} \operatorname{Hom}_{A}\left(M \otimes U_{1,2}, N\right)$ for each pair of simple modules $M, N$. This gives the $E_{6}$ diagram, as expected,

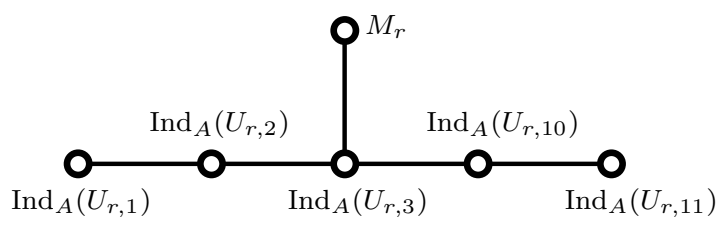


It is also easy to see that the simple $A$-modules consist of the following irreducible $M_{5,12}$ representations,

$$
\begin{array}{ll}
\operatorname{Ind}_{A}\left(U_{r, 1}\right)=U_{r, 1} \oplus U_{r, 7} & \operatorname{Ind}_{A}\left(U_{r, 10}\right)=U_{r, 4} \oplus U_{r, 6} \oplus U_{r, 10} \\
\operatorname{Ind}_{A}\left(U_{r, 2}\right)=U_{r, 2} \oplus U_{r, 6} \oplus U_{r, 8} & \operatorname{Ind}_{A}\left(U_{r, 11}\right)=U_{r, 5} \oplus U_{r, 11} \\
\operatorname{Ind}_{A}\left(U_{r, 3}\right)=U_{r, 3} \oplus U_{r, 5} \oplus U_{r, 7} \oplus U_{r, 9} & M_{r}=U_{r, 4} \oplus U_{r, 8}
\end{array}
$$

The weights $\bmod \mathbb{Z}$ of these irreducible representations as given in the Kac table above then tell us which of these simple modules are local, and how they are identified with the irreducible representations of $M_{2,5} \times M_{3,4}$. One finds the following local, simple $A$-modules

$$
\begin{array}{lll}
\operatorname{Ind}_{A}\left(U_{1,1}\right)=\mathbf{1} \times \mathbf{1} & M_{1}=1 \times \sigma & \operatorname{Ind}_{A}\left(U_{1,11}\right)=1 \times \varepsilon \\
\operatorname{Ind}_{A}\left(U_{3,1}\right)=\phi \times \mathbf{1} & M_{3}=\phi \times \sigma & \operatorname{Ind}_{A}\left(U_{3,11}\right)=\phi \times \varepsilon .
\end{array}
$$

Next we turn to the action of topological defects in each of the two factors on the boundary conditions (C.15), i.e. the action of $X_{\phi} \times X_{1}, X_{1} \times X_{\sigma}$ and $X_{1} \times X_{\varepsilon}$. For $X_{\phi} \times X_{1}$, this action amounts to tensoring with the bimodule $\alpha_{A}\left(U_{3,1}\right)$, and one simply gets, for $N_{r}=\operatorname{Ind}_{A}\left(U_{r, s}\right)$ or $N_{r}=M_{r}$,

$$
\alpha_{A}\left(U_{3,1}\right) \otimes_{A} N_{1}=N_{3} \quad, \quad \alpha_{A}\left(U_{3,1}\right) \otimes_{A} N_{3}=N_{1} \oplus N_{3} .
$$

This explains the effect on the odd nodes of the $A_{4}$ diagram in $(6.3)$. The action of $X_{1} \times X_{\varepsilon}$ is equally easy to obtain. First of all, $\alpha_{A}\left(U_{1,11}\right) \otimes_{A} \operatorname{Ind}_{A}\left(U_{r, s}\right)=\operatorname{Ind}_{A}\left(U_{r, 12-s}\right)$. The action on $M_{r}$ can then be found by applying $\alpha_{A}\left(U_{1,11}\right) \otimes_{A}(-)$ to both sides of $\operatorname{Ind}_{A}\left(U_{r, 4}\right) \cong$ $\operatorname{Ind}_{A}\left(U_{r, 10}\right) \oplus M_{r}$, which then gives $\alpha_{A}\left(U_{1,11}\right) \otimes_{A} M_{r}=M_{r}$.

For the action of $M_{1}^{\text {bi }}$ (the bimodule obtained from the local module $M_{1}$ as in section C.1.4 one can compare the action of $\operatorname{Ind}_{A}\left(U_{1,4}\right)$ and $\operatorname{Ind}_{A}\left(U_{1,10}\right)$. For example,

$$
\begin{aligned}
& \alpha_{A}\left(U_{1,4}\right) \otimes_{A} \operatorname{Ind}_{A}\left(U_{r, 2}\right) \cong \operatorname{Ind}_{A}\left(U_{r, 11}\right) \oplus \operatorname{Ind}_{A}\left(U_{r, 3}\right) \oplus \operatorname{Ind}_{A}\left(U_{r, 3}\right) \\
& \alpha_{A}\left(U_{1,10}\right) \otimes_{A} \operatorname{Ind}_{A}\left(U_{r, 2}\right) \cong \operatorname{Ind}_{A}\left(U_{r, 11}\right) \oplus \operatorname{Ind}_{A}\left(U_{r, 3}\right)
\end{aligned}
$$

from which we conclude that $M_{1}^{\text {bi }} \otimes_{A} \operatorname{Ind}_{A}\left(U_{1,2}\right) \cong \operatorname{Ind}_{A}\left(U_{1,3}\right)$.

So far we have outlined how to obtain the data presented in figure (6.3). Let us now turn to the computation of the coefficients $\omega$. For the factorising boundary conditions, i.e. those corresponding to the modules (C.17), one has $\omega=1$. The remaining boundary conditions are related by the action of the topological defects, and hence it is enough to compute $\omega$ for one representative. We pick the module $\operatorname{Ind}_{A}\left(U_{1,2}\right)$, because in this case the simplified formula (C.8) is applicable. To evaluate it, we need

$$
\operatorname{dim} U_{1,2}=-\frac{\sqrt{2}}{1+\sqrt{3}}, \quad \operatorname{dim} U_{1,7}=2-\sqrt{3}, \quad s_{U_{1,2}, U_{1,7}}=\frac{\sqrt{2}}{1+\sqrt{3}} .
$$

The result is $\omega_{\operatorname{Ind}_{A}\left(U_{1,2}\right)}=-2-\sqrt{3}$. Inserting this value into (2.9) gives the reflection and transmission coefficients stated in (6.4). 


\section{C.4 Lee-Yang $\times \mathrm{M}_{2,7}$}

The final example of a product of minimal models that can be described as an extension of another minimal model is $M_{2,5} \times M_{2,7}$, which is equivalent to the $E_{8}$-invariant of $M_{7,30}$. The minimal model $M_{2,7}$ has central charge $c=-\frac{68}{7}$ and Kac table

\begin{tabular}{c||c|c|c|c|c|c|}
\multicolumn{1}{c}{$M_{2,7}$} \\
\hline 1 & 0 & $-\frac{2}{7}$ & $-\frac{3}{7}$ & $-\frac{3}{7}$ & $-\frac{2}{7}$ & 0 \\
\hline \hline & 1 & 2 & 3 & 4 & 5 & 6
\end{tabular}

Let us denote the irreducible representation of weight $-\frac{2}{7}$ by $\alpha$ and that of weight $-\frac{3}{7}$ by $\beta$. The fusion rules are then $\alpha \otimes \alpha=\mathbf{1} \oplus \beta, \alpha \otimes \beta=\alpha \oplus \beta$ and $\beta \otimes \beta=\mathbf{1} \oplus \alpha \oplus \beta$.

We will not state the Kac table of $M_{7,30}$ explicitly. The relevant algebra is built on the representation $A=U_{1,1} \oplus U_{1,11} \oplus U_{1,19} \oplus U_{1,29}$ whose irreducible summands have highest weights $0,2,12$ and 35, respectively. In particular, the chiral field $W$ is a highest weight state in $U_{W}=U_{1,11}$. Note that $J \equiv U_{1,29}$ is a simple current which acts under fusion as $J \otimes U_{r, s} \cong U_{r, 30-s}$. Since $A \otimes J \cong A$ it is not hard to convince oneself that for induced modules,

$$
\operatorname{Ind}_{A}(U) \cong \operatorname{Ind}_{A}(J \otimes U)
$$

It is thus enough to consider the induced modules for $U_{r, s}$ with $s \in\{1,2, \ldots, 15\}$. These induced modules decompose as follows into simple modules

$$
\begin{array}{lll}
\left.\operatorname{Ind}_{A}\left(U_{r, 1}\right) \text { (simple }\right) & \operatorname{Ind}_{A}\left(U_{r, 9}\right) \cong \operatorname{Ind}_{A}\left(U_{r, 3}\right) \oplus \operatorname{Ind}_{A}\left(U_{r, 5}\right) \\
\left.\operatorname{Ind}_{A}\left(U_{r, 2}\right) \text { (simple }\right) & \operatorname{Ind}_{A}\left(U_{r, 10}\right) \cong \operatorname{Ind}_{A}\left(U_{r, 2}\right) \oplus \operatorname{Ind}_{A}\left(U_{r, 4}\right) \oplus M_{r}^{(2)} \\
\left.\operatorname{Ind}_{A}\left(U_{r, 3}\right) \text { (simple }\right) & \operatorname{Ind}_{A}\left(U_{r, 11}\right) \cong \operatorname{Ind}_{A}\left(U_{r, 1}\right) \oplus \operatorname{Ind}_{A}\left(U_{r, 3}\right) \oplus \operatorname{Ind}_{A}\left(U_{r, 5}\right) \\
\operatorname{Ind}_{A}\left(U_{r, 4}\right) \quad(\text { simple }) & \operatorname{Ind}_{A}\left(U_{r, 12}\right) \cong \operatorname{Ind}_{A}\left(U_{r, 2}\right) \oplus \operatorname{Ind}_{A}\left(U_{r, 4}\right) \oplus M_{r}^{(1)} \\
\operatorname{Ind}_{A}\left(U_{r, 5}\right) \text { (simple) } & \operatorname{Ind}_{A}\left(U_{r, 13}\right) \cong \operatorname{Ind}_{A}\left(U_{r, 3}\right) \oplus \operatorname{Ind}_{A}\left(U_{r, 5}\right) \oplus M_{r}^{\phi} \\
\operatorname{Ind}_{A}\left(U_{r, 6}\right) \cong M_{r}^{(1)} \oplus M_{r}^{(2)} & \operatorname{Ind}_{A}\left(U_{r, 14}\right) \cong \operatorname{Ind}_{A}\left(U_{r, 4}\right) \oplus M_{r}^{(1)} \oplus M_{r}^{(2)} \\
\operatorname{Ind}_{A}\left(U_{r, 7}\right) \cong \operatorname{Ind}_{A}\left(U_{r, 5}\right) \oplus M_{r}^{\phi} & \operatorname{Ind}_{A}\left(U_{r, 15}\right) \cong 2 \operatorname{Ind}_{A}\left(U_{r, 5}\right) \\
\operatorname{Ind}_{A}\left(U_{r, 8}\right) \cong \operatorname{Ind}_{A}\left(U_{r, 4}\right) \oplus M_{r}^{(1)} &
\end{array}
$$

Here $r$ takes values in $\{1,3,5\}$. There are thus 24 simple $A$-modules, namely $\operatorname{Ind}_{A}\left(U_{r, s}\right)$ with $s \in\{1,2,3,4,5\}$, as well as $M_{r}^{(1)}, M_{r}^{(2)}$ and $M_{r}^{\phi}$. As before one can find the decomposition of these simple modules into representations of $M_{7,30}$, and by evaluating the conformal weight modulo $\mathbb{Z}$ one finds that there are six local modules. Their identification with 
representations of the product model is as follows

$$
\begin{array}{lll}
\operatorname{Ind}_{A}\left(U_{1,1}\right)=\mathbf{1} \times \mathbf{1} & \operatorname{Ind}_{A}\left(U_{3,1}\right)=\beta \times \mathbf{1} & \operatorname{Ind}_{A}\left(U_{5,1}\right)=\alpha \times \mathbf{1} \\
M_{1}^{\phi}=\mathbf{1} \times \phi & M_{3}^{\phi}=\beta \times \phi & M_{5}^{\phi}=\alpha \times \phi
\end{array}
$$

To obtain the $E_{8}$ diagram we need to compute the effect of tensoring the simple modules with $U_{1,2}$ from the right. For induced modules this is again an easy exercise. For the simple modules not isomorphic to induced modules one can, for example, tensor both sides of $\operatorname{Ind}_{A}\left(U_{r, 7}\right) \cong \operatorname{Ind}_{A}\left(U_{r, 5}\right) \oplus M_{r}^{\phi}$ from the right with $U_{1,2}$ which gives

$$
\operatorname{Ind}_{A}\left(U_{r, 6}\right) \oplus \operatorname{Ind}_{A}\left(U_{r, 8}\right) \cong \operatorname{Ind}_{A}\left(U_{r, 4}\right) \oplus \operatorname{Ind}_{A}\left(U_{r, 6}\right) \oplus\left(M_{r}^{\phi} \otimes U_{1,2}\right)
$$

Substituting the decompositions in (C.22) it follows that $M_{r}^{\phi} \otimes U_{1,2} \cong M_{r}^{(1)}$. For $M_{r}^{(1)}$ and $M_{r}^{(2)}$ one can proceed similarly. Altogether one finds, for $r \in\{1,3,5\}$,

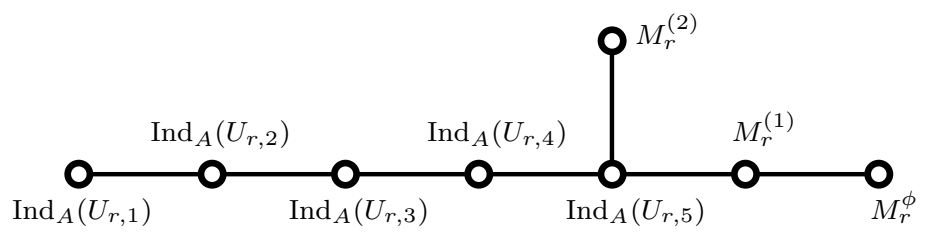

The action of the topological defects $X_{1} \times X_{\alpha}, X_{1} \times X_{\beta}$ and $X_{\phi} \times 1$ on these conformal boundary conditions corresponds to tensoring the corresponding $A$-modules over $A$ from the left with $\alpha_{A}\left(U_{5,1}\right), \alpha_{A}\left(U_{3,1}\right)$, and $M_{1}^{\phi, \text { bi }}$, respectively. To compute these tensor products, it is easiest to work with isomorphism classes rather than directly with modules and bimodules. In this way we obtain the fusion ring of bimodules, and a representation of that ring on the $\mathbb{Z}$-module generated by the isomorphism classes of simple modules. The point is that in this setting it makes sense to consider differences. For a bimodule $B$ or a module $M$, denote by $[B]$ and $[M]$ the corresponding isomorphism classes. Then

$$
\begin{aligned}
& {\left[M_{1}^{\phi, \mathrm{bi}}\right]=\left[\alpha_{A}\left(U_{1,7}\right)\right]-\left[\alpha_{A}\left(U_{1,5}\right)\right],} \\
& {\left[M_{r}^{(1)}\right]=\left[\operatorname{Ind}_{A}\left(U_{1,8}\right)\right]-\left[\operatorname{Ind}_{A}\left(U_{1,4}\right)\right], \quad\left[M_{r}^{\phi}\right]=\left[\operatorname{Ind}_{A}\left(U_{1,7}\right)\right]-\left[\operatorname{Ind}_{A}\left(U_{1,5}\right)\right],} \\
& {\left[M_{r}^{(2)}\right]=\left[\operatorname{Ind}_{A}\left(U_{1,6}\right)\right]-\left[\operatorname{Ind}_{A}\left(U_{1,8}\right)\right]+\left[\operatorname{Ind}_{A}\left(U_{1,4}\right)\right] .}
\end{aligned}
$$

One can now compute, for example,

$$
\begin{aligned}
& {\left[M_{1}^{\phi, \mathrm{bi}} \otimes_{A} \operatorname{Ind}_{A}\left(U_{r, 2}\right)\right]=\left[M_{1}^{\phi, \mathrm{bi}}\right] \cdot\left[\operatorname{Ind}_{A}\left(U_{r, 2}\right)\right]} \\
& =\left[\alpha_{A}\left(U_{1,7}\right)\right] \cdot\left[\operatorname{Ind}_{A}\left(U_{r, 2}\right)\right]-\left[\alpha_{A}\left(U_{1,5}\right)\right] \cdot\left[\operatorname{Ind}_{A}\left(U_{r, 2}\right)\right] \\
& =\left[\alpha_{A}\left(U_{1,7}\right) \otimes_{A} \operatorname{Ind}_{A}\left(U_{r, 2}\right)\right]-\left[\alpha_{A}\left(U_{1,5}\right) \otimes_{A} \operatorname{Ind}_{A}\left(U_{r, 2}\right)\right] \\
& =\left[\operatorname{Ind}_{A}\left(U_{r, 6}\right) \oplus \operatorname{Ind}_{A}\left(U_{r, 8}\right)\right]-\left[\operatorname{Ind}_{A}\left(U_{r, 4}\right) \oplus \operatorname{Ind}_{A}\left(U_{r, 6}\right)\right] \\
& =\left[\operatorname{Ind}_{A}\left(U_{r, 6}\right)\right]+\left[\operatorname{Ind}_{A}\left(U_{r, 8}\right)\right]-\left[\operatorname{Ind}_{A}\left(U_{r, 4}\right)\right]-\left[\operatorname{Ind}_{A}\left(U_{r, 6}\right)\right]=\left[M_{r}^{(1)}\right]
\end{aligned}
$$


i.e. $M_{1}^{\phi, \text { bi }} \otimes_{A} \operatorname{Ind}_{A}\left(U_{r, 2}\right) \cong M_{r}^{(1)}$. Proceeding along these lines, one finds the action of the topological defects as given in (6.5).

Finally, we need to calculate the coefficients $\omega$ for the various conformal defects corresponding to the folded boundary conditions of the folded model. Due to the action of topological defects as given in (6.5), by comparing to (C.25) we see that it is enough to compute $\omega$ for the representatives $\operatorname{Ind}_{A}\left(U_{1, s}\right)$ with $s \in\{1,2,3,4\}$. Since all of these are induced modules, we can again apply (C.8). The following constants are needed,

$$
\begin{aligned}
& \operatorname{dim}\left(U_{1, s}\right)=(-1)^{s-1} \sin \left(\pi \frac{7 s}{30}\right) / \sin \left(\pi \frac{7}{30}\right), \\
& s_{U_{W}, U_{1, s}}=(-1)^{s-1} \sin \left(\pi \frac{77 s}{30}\right) / \sin \left(\pi \frac{7}{30}\right) .
\end{aligned}
$$

Substituting these into (C.8) one recovers (6.6).

\section{References}

[1] M. Oshikawa and I. Affleck, Defect Lines in the Ising Model and Boundary States on Orbifolds, Phys. Rev. Lett. 77 (1996) 2604 [hep-th/9606177].

[2] M. Oshikawa and I. Affleck, Boundary conformal field theory approach to the critical two-dimensional Ising model with a defect line, Nucl. Phys. B 495 (1997) 533 [cond-mat/9612187].

[3] E. Wong and I. Affleck, Tunneling in quantum wires: A Boundary conformal field theory approach, Nucl. Phys. B 417 (1994) 403.

[4] C. Chamon, M. Oshikawa and I. Affleck, Junctions of three quantum wires and the dissipative Hofstadter model, Phys. Rev. Lett. 91 (2003) 206403 [cond-mat/0305121].

[5] M. Oshikawa, C. Chamon and I. Affleck, Junctions of three quantum wires, J. Stat. Mech. 0602 (2006) P008 [cond-mat/0509675].

[6] D. Friedan, Entropy flow in near-critical quantum circuits, cond-mat/0505084.

[7] D. Friedan, Entropy flow through near-critical quantum junctions, cond-mat/0505085.

[8] C. Bachas, J. de Boer, R. Dijkgraaf and H. Ooguri, Permeable conformal walls and holography, JHEP 0206 (2002) 027 [hep-th/0111210].

[9] V.B. Petkova and J.B. Zuber, Generalised twisted partition functions, Phys. Lett. B 504 (2001) 157 [hep-th/0011021].

[10] V.B. Petkova and J.B. Zuber, The many faces of Ocneanu cells, Nucl. Phys. B 603 (2001) 449 [hep-th/0101151].

[11] C.H.O. Chui, C. Mercat, W.P. Orrick and P.A. Pearce, Integrable lattice realizations of conformal twisted boundary conditions, Phys. Lett. B 517 (2001) 429 [hep-th/0106182].

[12] R. Coquereaux and G. Schieber, Twisted partition functions for ADE boundary conformal field theories and Ocneanu algebras of quantum symmetries, J. Geom. Phys. 42 (2002) 216 [hep-th/0107001].

[13] T. Quella and V. Schomerus, Symmetry breaking boundary states and defect lines, JHEP 0206 (2002) 028 [hep-th/0203161]. 
[14] J. Fuchs, I. Runkel and C. Schweigert, TFT construction of RCFT correlators. I: Partition functions, Nucl. Phys. B 646 (2002) 353 [hep-th/0204148].

[15] K. Graham and G.M.T. Watts, Defect lines and boundary flows, JHEP 0404 (2004) 019 [hep-th/0306167].

[16] J. Fröhlich, J. Fuchs, I. Runkel and C. Schweigert, Kramers-Wannier duality from conformal defects, Phys. Rev. Lett. 93 (2004) 070601 [cond-mat/0404051].

[17] J. Fröhlich, J. Fuchs, I. Runkel and C. Schweigert, Duality and defects in rational conformal field theory, Nucl. Phys. B 763 (2007) 354 [hep-th/0607247].

[18] J. Fuchs and C. Schweigert, Symmetry breaking boundaries. I: General theory, Nucl. Phys. B $\mathbf{5 5 8}$ (1999) 419 [hep-th/9902132].

[19] A. Recknagel, Permutation branes, JHEP 0304 (2003) 041 [hep-th/0208119].

[20] T. Quella, On the hierarchy of symmetry breaking D-branes in group manifolds, JHEP 0212 (2002) 009 [hep-th/0209157].

[21] S. Fredenhagen and T. Quella, Generalised permutation branes, JHEP 0511 (2005) 004 [hep-th/0509153].

[22] S. Fredenhagen and M.R. Gaberdiel, Generalised $N=2$ permutation branes, JHEP 0611 (2006) 041 [hep-th/0607095].

[23] V.B. Petkova and J.B. Zuber, Conformal boundary conditions and what they teach us in: Non-perturbative QFT Methods and Their Applications, Z. Horvath and L. Palla, eds. (World Scientific, Singapore 2001), p.1 [hep-th/0103007].

[24] J.L. Cardy, Boundary Conditions, Fusion Rules And The Verlinde Formula, Nucl. Phys. B 324 (1989) 581.

[25] T. Quella and V. Schomerus, Asymmetric cosets, JHEP 0302 (2003) 030 [hep-th/0212119].

[26] I. Brunner and M.R. Gaberdiel, Matrix factorisations and permutation branes, JHEP 0507 (2005) 012 [hep-th/0503207].

[27] C. Caviezel, S. Fredenhagen and M.R. Gaberdiel, The RR charges of A-type Gepner models, JHEP 0601 (2006) 111 [hep-th/0511078].

[28] I. Affleck and A.W.W. Ludwig, Universal noninteger 'ground state degeneracy' in critical quantum systems, Phys. Rev. Lett. 67 (1991) 161.

[29] J.L. Cardy, Conformal Invariance And Surface Critical Behavior, Nucl. Phys. B 240 (1984) 514.

[30] A. Fring and R. Köberle, Factorized scattering in the presence of reflecting boundaries, Nucl. Phys. B 421 (1994) 159 [hep-th/9304141].

[31] S. Ghoshal and A.B. Zamolodchikov, Boundary S matrix and boundary state in two-dimensional integrable quantum field theory, Int. J. Mod. Phys. A 9 (1994) 3841 [Erratum-ibid. A 9 (1994) 4353] [hep-th/9306002].

[32] G. Delfino, G. Mussardo and P. Simonetti, Statistical models with a line of defect, Phys. Lett. B 328 (1994) 123 [hep-th/9403049].

[33] G. Delfino, G. Mussardo and P. Simonetti, Scattering theory and correlation functions in statistical models with a line of defect, Nucl. Phys. B 432 (1994) 518 [hep-th/9409076]. 
[34] R. Konik and A. LeClair, Purely transmitting defect field theories, Nucl. Phys. B 538 (1999) 587 [hep-th/9703085].

[35] A. LeClair and A.W.W. Ludwig, Minimal models with integrable local defects, Nucl. Phys. B 549 (1999) 546 [hep-th/9708135].

[36] P. Bowcock, E. Corrigan and C. Zambon, Affine Toda field theories with defects, JHEP 0401 (2004) 056 [hep-th/0401020].

[37] C. Bachas and M.R. Gaberdiel, Loop operators and the Kondo problem, JHEP 0411 (2004) 065 [hep-th/0411067].

[38] J. Fröhlich, J. Fuchs, I. Runkel and C. Schweigert, Picard groups in rational conformal field theory, Contemp. Math. 391 (2005) 85 [math.ct/0411507].

[39] B.M. McCoy and J.H.H. Perk, Two Spin Correlation Functions Of An Ising Model With Continuous Exponents, Phys. Rev. Lett. 44 (1980) 840.

[40] M. Henkel and A. Patkos, Critical exponents of defected Ising models and the U(1) Kac-Moody-Virasoro algebras, Nucl. Phys. B 285 (1987) 29.

[41] D. Friedan, The space of conformal boundary conditions for the $c=1$ Gaussian model, private notes (1999), The space of conformal boundary conditions for the $c=1$ Gaussian model (more), private notes (2003), both available from http://www.physics.rutgers.edu/ ${ }^{\sim}$ friedan

[42] R.A. Janik, Exceptional boundary states at $c=1$, Nucl. Phys. B 618 (2001) 675 [hep-th/0109021].

[43] M.R. Gaberdiel and A. Recknagel, Conformal boundary states for free bosons and fermions, JHEP 0111 (2001) 016 [hep-th/0108238].

[44] A. Cappelli, C. Itzykson and J.B. Zuber, The ADE classification of minimal and $A_{1}^{(1)}$ conformal invariant theories, Commun. Math. Phys. 113 (1987) 1.

[45] R.E. Behrend, P.A. Pearce, V.B. Petkova and J.B. Zuber, On the classification of bulk and boundary conformal field theories, Phys. Lett. B 444 (1998) 163 [hep-th/9809097].

[46] R.E. Behrend, P.A. Pearce, V.B. Petkova and J.B. Zuber, Boundary conditions in rational conformal field theories, Nucl. Phys. B 570 (2000) 525 [Nucl. Phys. B 579 (2000) 707] [hep-th/9908036].

[47] A. Fring and C. Korff, Affine Toda field theories related to Coxeter groups of non-crystallographic type, Nucl. Phys. B 729 (2005) 361 [hep-th/0506226].

[48] P. Dorey, A. Pocklington, R. Tateo and G. Watts, TBA and TCSA with boundaries and excited states, Nucl. Phys. B 525 (1998) 641 [hep-th/9712197].

[49] P. Dorey, I. Runkel, R. Tateo and G. Watts, g-function flow in perturbed boundary conformal field theories, Nucl. Phys. B 578 (2000) 85 [hep-th/9909216].

[50] S. Fredenhagen and V. Schomerus, On boundary RG-flows in coset conformal field theories, Phys. Rev. D 67 (2003) 085001 [hep-th/0205011].

[51] S. Fredenhagen, Organizing boundary RG flows, Nucl. Phys. B 660 (2003) 436 [hep-th/0301229]. 
[52] G. Felder, J. Fröhlich, J. Fuchs and C. Schweigert, Correlation functions and boundary conditions in RCFT and three-dimensional topology, Compos. Math. 131 (2002) 189 [hep-th/9912239].

[53] J. Fuchs, I. Runkel and C. Schweigert, TFT construction of RCFT correlators. IV: Structure constants and correlation functions, Nucl. Phys. B 715 (2005) 539 [hep-th/0412290].

[54] J. Fjelstad, J. Fuchs, I. Runkel and C. Schweigert, TFT construction of RCFT correlators. V: Proof of modular invariance and factorisation, Theo. and Appl. of Cat. 16 (2006) 342 [hep-th/0503194].

[55] J. Fröhlich, J. Fuchs, I. Runkel and C. Schweigert, Correspondences of ribbon categories, Adv. Math. 199 (2006) 192 [math.ct/0309465]. 